\title{
Development of single- and two-tone responses of anteroventral cochlear nucleus neurons in gerbil ${ }^{1}$
}

\author{
Dawn L. Konrad-Martin ${ }^{\text {b }}$, Rudolf Rübsamen ${ }^{2, b}$, Gerd J. Dörrscheidt ${ }^{3, b}$, \\ Edwin W. Rubel ${ }^{\mathrm{a}, *}$ \\ a Virginia Merrill Bloedel Hearing Research Center, Department of Otolaryngology-HNS, Box 357923, University of Washington School of Medicine, \\ Seattle, WA 98195-7923, USA \\ b Department of Speech and Hearing Sciences, University of Washington, Seattle, WA 98195, USA
}

Received 27 September 1997; revised 26 March 1998; accepted 31 March 1998

\begin{abstract}
Responses of anteroventral cochlear nucleus (AVCN) neurons in developing gerbils were obtained to single-tone stimuli, and twotone stimuli elicited by best frequency probes presented over a range of intensities. Neurons displayed Type I, Type I/III, and Type III receptive field patterns. Best frequencies ranged from 1.5 to $10.0 \mathrm{kHz}$. Two-tone suppression (2TS) was first observed in 5 of 16 neurons examined at $14 \mathrm{dab}$, and in all neurons examined in gerbils aged 15 to $60 \mathrm{dab}$. Suppression areas grew larger, and discharge rate reductions became greater with age. Features of the two-tone responses that were highly correlated with single-tone responses across age groups include maximum rate reductions and suppression area thresholds. The intensity level of the CF probe-tone also influenced these features of 2TS. Maximum rate reductions to below spontaneous rate levels of activity were common across age groups. Results suggest that the cochlear amplifier is present and fundamentally adult-like by 15 dab for the regions of the cochlea coding the mid frequencies in gerbil. Over the subsequent week, contributions to the developing two-tone responses by the cochlear amplifier increase slightly. Two-tone responses are influenced by central inhibitory mechanisms as early as 14 dab. (C) 1998 Elsevier Science B.V. All rights reserved.
\end{abstract}

Key words: Development; Cochlear nuclear complex; Two-tone suppression

\section{Introduction}

Two-tone suppression (2TS) can be operationally defined as the decrease in activity evoked by one tone (the probe) that occurs when it is presented together with a second tone (the suppressor). Suppression in mammals depends on the presence and correct functioning of the outer hair cells. It is thought to be generated by the

\footnotetext{
* Corresponding author. Tel.: +1 (206) 543-8360;

Fax: +1 (206) 616-1828; E-mail: rubel@u.washington.edu

2 Present address: Arbeitsgruppe Neurobiologie, Institut für Zoologie, Talstraße 33, 04103 Leipzig, Germany.

${ }^{3}$ Present address: Lehrstuhl für Allgemeine Zoologie und Neurobiologie, Ruhr-Universität, 48780 Bochum, Germany.

${ }^{1}$ Portions of this paper were presented at the Mid-winter Meeting of the Association for Research in Otolaryngology, February, 1994.
}

normal operating characteristics of physiologically active cochlear processes (Dallos et al., 1980; Schmiedt et al., 1980; Schmiedt and Zwislocki, 1980; Mills and Schmiedt, 1983; Patuzzi and Robertson, 1988). These processes, collectively termed the cochlear amplifier, are also responsible for the high degree of sensitivity and frequency tuning exhibited by the healthy cochlea.

Cochlear responses to single- and two-tone stimuli are intrinsically linked. The cochlear amplifier boosts vibratory responses, primarily to low level stimuli, near the basilar membrane place which codes the input frequency (e.g. Neely and Kim, 1983, Neely and Kim, 1986; Yates et al., 1992). It is thought that suppression of a given probe-tone's response takes place where the suppressor-tone itself engages and saturates the cochlear amplifier in the probe's characteristic frequency $(\mathrm{CF})$ region, rendering it unavailable to amplify probe in- 
duced activity (Geisler et al., 1990). Therefore, it is hypothesized that $2 \mathrm{TS}$ can be used to infer the contribution of the cochlear amplifier to probe-driven discharge activity.

Cochlear sensitivity and tuning improve postnatally in altricial mammals (for review, see Rübsamen, 1992; Walsh and Romand, 1992; Rübsamen and Lippe, 1998). In the Mongolian gerbil (Meriones unguiculatus), auditory responses are first able to be observed at 12 days after birth (dab) using cochlear microphonic techniques (Woolf and Ryan, 1984). Adult-like sensitivity and tuning is reached in the majority of auditory nerve fibers and cochlear nucleus neurons by approximately 18 to 21 dab (Woolf and Ryan, 1985; Echteler et al., 1989).

In addition to changes in cochlear function in altricial animals, postnatal alterations in cochlear structures have also been observed in a variety of species (for review see Rubel, 1978; Walsh and Romand, 1992). Certain of these changes could affect cochlear function through alterations of basilar membrane mass and stiffness characteristics. Other changes, including a dramatic increase in the endocochlear potential (cat: Fernandez and Hinojosa, 1974; gerbil: Woolf et al., 1986; McGuirt et al., 1995; rat: Bosher and Warren, 1971), are thought to influence cochlear response properties through changes in the processes underlying the cochlear amplifier.

The development of those cochlear response properties which are likely to be produced primarily by active cochlear processes has been examined extensively using distortion product otoacoustic emissions (DPOAEs) (gerbil: Norton et al., 1991; Mills et al., 1994, Mills and Rubel, 1996; rat: Henley et al., 1989; Lenoir and Puel, 1987), and less often using 2TS (cat: Fitzakerley et al., 1994a, Fitzakerley et al., 1994b). These studies have shown that the cochlear amplifier does, in fact, mature postnatally in altricial animals. However, the relationship between the ontogeny of the cochlear amplifier and the maturation of cochlear sensitivity and tuning has not been fully characterized.

The present study has two parts. The first investigates the development of responses elicited by twotone stimulation of mid frequency primary-like neurons in the AVCN. Relationships between features of 2TS and neuronal response properties are examined at various developmental time points. Both the rate reduction produced by high level suppressors (2TS magnitude) and the shapes of suppression areas (2TS area thresholds and bandwidths) are examined. Data from the only other investigations of the development of two-tone rate suppression (Fitzakerley et al., 1994a, Fitzakerley et al., 1994b) showed a large amount of variability which obscured any systematic relationship between emerging neuronal tip regions and the ontogeny of 2TS. It was therefore a goal in the present study to ensure that maximum 2TS was obtained by using a broad range of suppressor- and probe-tone combinations.

The second part of this study attempts to relate some of the observed age-related changes in 2TS to changes in the amount of $\mathrm{CF}$ amplification supplied to the probe-tone's response, and to provide some insight into possible contributions to the AVCN two-tone response by neural inhibition. Suppression is examined over a range of probe-tone intensity levels. Comparisons are made between effects on 2TS caused by changes in age and in the level of the probe. It was hypothesized that for a given neuron, increasing the level of the probe would cause a systematic reduction in the amplification of the probe's basilar membrane response (e.g. Rhode, 1978, Ruggero and Rich, 1991) and thus reduce cochlear 2TS (Ruggero et al., 1992). On the other hand, we propose that increasing the probe intensity might cause an increase in contributions to the two-tone responses by cochlear nucleus inhibition.

\section{Materials and methods}

\subsection{Animal preparation}

Thirty Mongolian gerbils (Meriones unguiculatus) aged 13 to 60 days after birth (dab) were studied. Animals were divided into five age groups based on endocochlear potential (EP) development in gerbil (Woolf et al., 1986; McGuirt et al., 1995): 13 dab, 14 dab, 15 to $17 \mathrm{dab}, 18$ to $21 \mathrm{dab}$, and 23 to $60 \mathrm{dab}$. Table 1 provides the numbers of animals and neurons in each age group, as well as other pertinent information.

Gerbils were purchased from a commercial supplier (Tumblebrook Farms, Brookfield, MA), or raised in the University of Washington breeding colony, originally obtained from the same supplier. Breeding pairs were checked daily for new litters and the day of birth was considered dab 0. Large litters were culled to six. Animal care was in accordance with the guidelines of the University of Washington Animal Care Committee reviewed under NIH grant DC00395.

Surgery, stimulus delivery and response recording were performed in a double-walled sound attenuated room (Industrial Acoustics Corporation). Animals were anaesthetized with a ketamine hydrochloride (Ketaset; $15 \mathrm{mg} / \mathrm{kg}$ ) and xylazine (Rompun; $5 \mathrm{mg} / \mathrm{kg}$ ) mixture administered intraperitoneally (IP). Supplemental doses were infused subcutaneously approximately every 45 min to maintain a surgical anesthetic level. The animal's core body temperature was measured by a rectal thermometer and maintained at approximately $37.5^{\circ} \mathrm{C}$.

The dorsal aspect of the animal's skull was surgically exposed. A bolt was glued in a midline position and 
stabilized with dental cement. The bolt secured the head to a custom holder that oriented the animal by discrete degree increments in the coronal and sagittal planes. A glass micropipette electrode housing a silver chloride wire bathed in $3 \mathrm{M} \mathrm{KCl}$ was attached to a Newport piezoelectric micromanipulator and lowered into the brain through a burr hole approximately 2500-3000 $\mu \mathrm{m}$ caudal to the lambda suture. The reference electrode was placed in the cerebellum.

\subsection{Procedure}

Recordings were made in the AVCN. This was considered optimal to routinely achieve the minimal cell holding times required for our protocol $(18 \mathrm{~min})$ in developing gerbils, which have an incompletely ossified skull, and therefore cannot be fixed as rigidly as would be necessary for similar duration recordings from individual auditory nerve fibers. Average cell holding times were in excess of $30 \mathrm{~min}$, enabling us to present five runs each of a large array of suppressor-tone intensity/frequency combinations (up to 256) for as many as 8 probe levels per neuron. To maximize the number of suppressor stimuli used to characterize a given suppression area, 2TS was examined for suppressor-tones higher in frequency than the excitatory characteristic frequency $(\mathrm{CF})$ whereas below $\mathrm{CF}$ suppression areas were usually ignored.

Prior to obtaining the data reported herein, the position of the AVCN, as well as a multiunit tonotopic map of the area, was determined for each animal. This was done using a medium impedance (ca. $10 \mathrm{~m} \Omega$ ) microelectrode and approximately eight electrode penetrations. Tone pips swept in frequency and intensity were presented in a near field stimulation paradigm. Penetrations were initially aimed at the cochlear nucleus using stereotaxic techniques. Successive recordings were varied systematically in their rostrocaudal and mediolateral orientation. Multiunit responsiveness was acquired each $100 \mu \mathrm{m}$ in electrode depth between 4000 and $6000 \mu \mathrm{m}$ measured from the dorsal brain surface. The coordinates for the AVCN were verified by the rostral to caudal and medial to lateral, low to high frequency gradient typically found in the AVCN, which is inverse to the caudal to rostral frequency gradient observed in the posteroventral cochlear nucleus (PVCN). After establishing the exact individual-specific stereotaxic coordinates for recording from a specific frequency domain within the AVCN, the medium impedance electrode was replaced by a high impedance electrode (20-30 $\mathrm{m} \Omega$ ) for use in data collection. In a number of animals the electrode location was also validated by making alcian blue or fast green deposits in the region presumed to be AVCN. In all cases the dye mark was within the spherical bushy cell region of the AVCN. Ten of the neurons included in this study had a clear prepotential response, which preceded a second spike of larger amplitude. Prepotentials are thought to reflect changes in the membrane potential of the large auditory nerve endings, endbulbs of Held (Pfeiffer, 1966), and were taken as further verification that the recording position was indeed the spherical bushy cell region of AVCN. At the end of the experiments, animals were deeply anaesthetized using Nembutal $(50 \mathrm{mg} / 100 \mathrm{~g}$ body weight).

\subsection{Acoustic system and calibration}

Single tones presented alone, and suppressor-tones presented in two-tone stimulus conditions, were digitally synthesized by a Compaq (386/20) microcomputer equipped with a 12-bit D/A converter (Neuroboard; custom made). For two-tone stimulus conditions, a second channel was utilized for the probe-tone. The probe was produced by a tone burst generator and its frequency was manually selected by the experimenter. The sound pressure levels of the two acoustic stimuli were controlled independently. The two signals were electrically mixed, passed through an anti-aliasing filter, and amplified by a low distortion amplifier (Crown International 150A). The intensities of the acoustic stimuli were corrected for the response of the stimulus transducer and were delivered to the animal's right ear via a funnel shaped adapter attached to a dynamic earphone (Beyerdynamic DT-48) positioned in the ear canal approximately $3 \mathrm{~mm}$ from the ear drum. The maximum output of the speakers in the corrected mode was 105 $\mathrm{dB}$ SPL. All tones were $100 \mathrm{~ms}$ in duration with $5 \mathrm{~ms}$ rise/fall times, and usually presented with $100 \mathrm{~ms}$ interstimulus intervals. In 13-14 dab animals it was necessary to increase the interstimulus intervals to 200 or 300 $\mathrm{ms}$ in order to get consistent stimulus-driven responses. For $13 \mathrm{dab}$ animals, where thresholds were above 105 dB SPL, the loudspeaker correction was bypassed to achieve a maximum presentation level of $125 \mathrm{~dB}$ SPL at $3.5 \mathrm{kHz}$.

\subsection{Data acquisition and analysis}

From each single isolated unit recorded in the AVCN, we first obtained responses to single-tone stimulus conditions. The purpose of the initial recording was to detect the presence of inhibitory side-bands. For this series of recordings, a broad frequency range was chosen (which necessarily limited the frequency resolution of the recording to about 4 frequencies per octave). A second series of recordings was then made which spanned only the excitatory area, using finer frequency increments (6 to 10 frequencies per octave). Data presented for single-tone stimulus conditions were obtained from the latter array of responses.

An example of the data acquisition and analysis pro- 
cedure for single-tone stimulus conditions is given in Fig. 1. Panel A shows a set of excitatory area responses recorded from an AVCN neuron in a gerbil with adult hearing thresholds ( $30 \mathrm{dab})$. The boundaries of neuronal excitatory areas were generated from the spike information using an algorithm described previously by Rübsamen et al. (1995). The algorithm determines increases or decreases in discharge activity relative to a baseline response rate, using a $90 \%$ confidence level. The baseline response rate for single-tone conditions is determined from activity collected during runs in which each of the tones was presented at sub-threshold

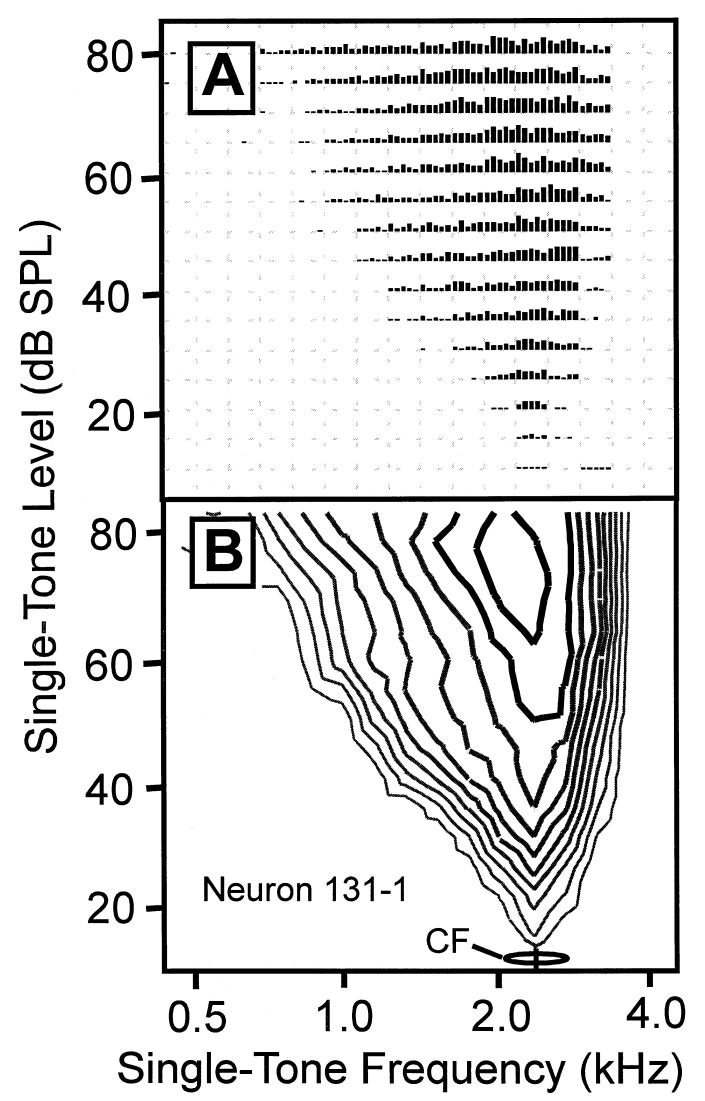

Fig. 1. Excitatory response area. A set of analyzed responses to single tones recorded from an AVCN neuron in a gerbil with mature auditory thresholds $(30 \mathrm{dab})$. Sixteen frequencies between 0.5 to 4.2 $\mathrm{kHz}$ were presented at 15 intensity levels (10 to $80 \mathrm{~dB}$ SPL), and each frequency/intensity combination was repeated five times. A: The relative number of spikes evoked by each stimulus is indicated by the height of the bars. The spontaneous firing rate was calculated from discharge activity collected when tones were presented at a sub-threshold level of $10 \mathrm{~dB}$ SPL. B: An algorithm was used to calculate statistically significant increases (and decreases) in neuronal activity relative to the baseline taken from the spontaneous activity. The neuron's excitatory area is delineated by the outermost iso-response contour, which encloses those stimulus conditions resulting in a significant increase in neuronal discharges above the baseline ( $90 \%$ confidence level). Each successive contour line represents stimulus conditions evoking significant increases in discharge activity above that produced by the stimuli comprising the immediately preceding iso-response contour. The unit's CF is indicated. levels. The baseline discharge rate for the example shown in Fig. 1, was calculated from discharges measured when tones were presented at a sub-threshold level of $10 \mathrm{~dB}$ SPL. This neuron's excitatory area, shown in panel $\mathrm{B}$, is delineated by an iso-response contour which encloses those stimulus conditions resulting in an increase in neuronal discharges that reaches the upper level of the $90 \%$ confidence interval of baseline firing rate. The successive iso-response contours shown in Fig. $1 \mathrm{~B}$ were generated in an equivalent manner; each at the upper boundary of the previous $90 \%$ confidence interval. The entire response area is thereby transformed into a map of iso-response contours, which indicate the threshold curve, maximum responsiveness and spike rate versus level functions.

Discharge activity produced during a given stimulus condition was averaged and then used to determine neuronal discharge rates (in spikes per second). Spontaneous discharge rates were calculated by averaging the spike activity taken from a neuron's baseline activity level. For the majority of neurons described, 5 (100 msec) runs were presented at 16 frequencies, and spontaneous activity was measured over an $8 \mathrm{~s}$ period (interspersed in $100 \mathrm{msec}$ epochs throughout the data collection period).

After obtaining neuronal responses to single-tone stimuli, simultaneous two-tone stimulation was performed as follows. Probe-tones set at a fixed intensity and at the (excitatory) CF of the unit were simultaneously presented with computer-generated suppressortones, which varied in frequency and intensity. Fig. 2 shows an example of responses to two-tone stimulus conditions. Responses are for the same neuron presented in Fig. 1. For this example, the $2.3 \mathrm{kHz}$ probetone was fixed at a level of $20 \mathrm{~dB}$ SPL, and suppressortones were presented at 14 frequencies, ranging from 2.9 to $6.0 \mathrm{kHz}$, and at 16 intensities in the range of 20 to $95 \mathrm{~dB}$ SPL. The relative decrease in discharge activity caused by the suppressor-tone is represented in Fig. 2 by the reduction in the height of the bars relative to those generated by the probe-tone alone (in this case $2.3 \mathrm{kHz}$ at $20 \mathrm{~dB}$ SPL). The same statistical algorithm used to generate excitatory areas was used to produce the iso-suppression curve delineating the '2TS area', as shown in Fig. 2B by the dotted line. The 2TS area represents those stimulus conditions which produced discharge activity significantly decreased below the level of probe-tone induced excitation, again using a $90 \%$ confidence level. Frequency- and intensity-related differences in 2TS magnitude are indicated in Fig. 2B by the succession of contour lines shown, which represent consecutively greater magnitudes of $2 \mathrm{TS}$ that meet equivalent statistical criteria (spike rates below the $90 \%$ confidence interval of the next lower line on Fig. 2B). The best suppressor frequency (BSF) of the 2TS area is designated as that frequency which at the lowest inten- 


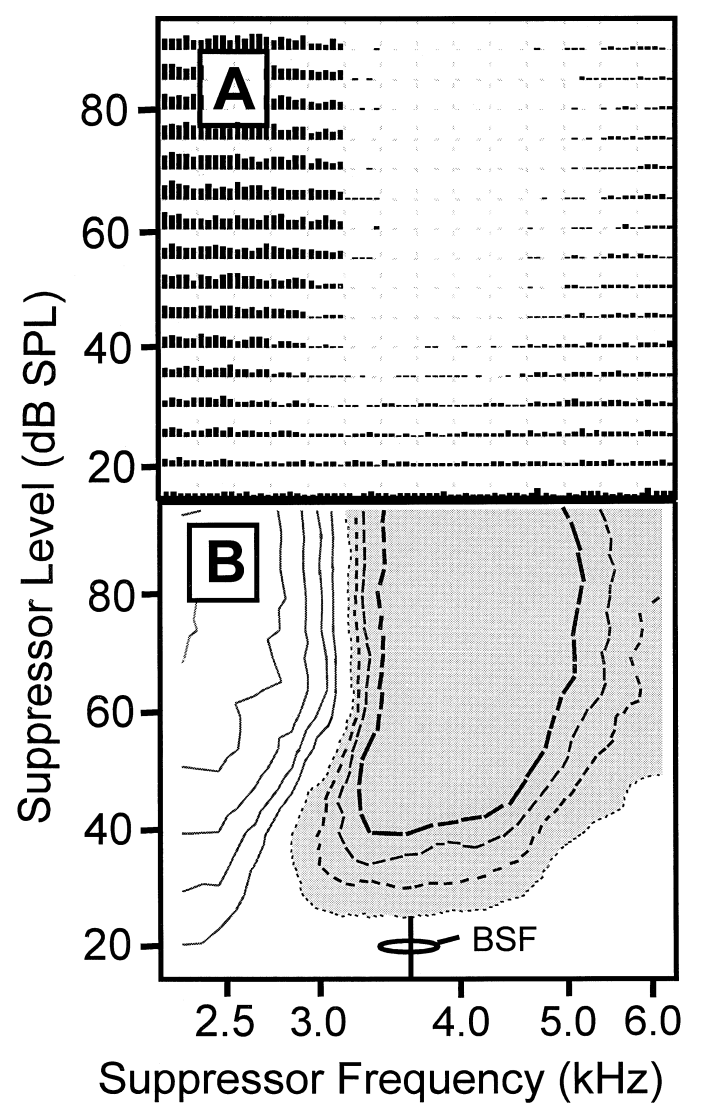

Fig. 2. Suppression side-band elicited by two-tone stimulation. Analyzed responses shown for the same neuron displayed in Fig. 1. The $2.3 \mathrm{kHz}$ probe was fixed at a level of $19 \mathrm{~dB}$ SPL, and suppressortones were presented at 14 frequencies ranging from 2.9 to $6.0 \mathrm{kHz}$, and at 16 intensities in the range of 20 to $95 \mathrm{~dB}$ SPL. A: The reduction in the height of the bars relative to those generated at the baseline (unaffected probe-tone response) represents the relative decrease in discharge activity caused by the suppressor-tone. B: The suppression or 2TS area (dotted line) is delineated by those stimulus conditions which produced discharge activity significantly decreased below the level of probe-tone induced excitation $(90 \%$ confidence level). Successive contour lines represent consecutively greater magnitudes of 2TS that meet equivalent statistical criteria. The best suppressor frequency (BSF) of the 2TS area is shown. The 2TS area Q10 is obtained using the bandwidth of the 2TS region measured at $10 \mathrm{~dB}$ above the $2 \mathrm{TS}$ threshold.

sity, produced 2TS. Its level denotes the 2TS threshold. The 2TS area Q10 is obtained using the bandwidth of the 2TS area measured at $10 \mathrm{~dB}$ above 2TS threshold.

Suppression recordings were first obtained using a CF probe-tone presented near threshold. While cell holding times lasted, additional $2 \mathrm{TS}$ recordings were made in which the probe level was varied in increments of 10 to $20 \mathrm{~dB}$, from $20 \mathrm{~dB}$ below to $70 \mathrm{~dB}$ above neuronal threshold. Several minutes of silence were presented between recordings in which high intensity probes were used. A given excitatory or 2TS area recording required approximately $4.5 \mathrm{~min}$ to complete.

\subsection{Off-line data analyses}

The reduction of probe-driven discharge activity within a 2TS area (2TS magnitude) was analyzed in several ways. Discharge rate reductions were calculated as absolute values (in spikes per second), and as the proportion of the probe-(alone)-driven discharge rate (in $\%$ re. single-tone responsiveness). For these measures, driven discharge activity generally includes spontaneous activity. Spontaneous discharge rates were subtracted from measures of driven discharge rates obtained under single- and two-tone conditions where it was desirable to view response reductions relative to the spontaneous activity level. For example, this manipulation of the data served as a crude assay for determining whether inhibition of spontaneous discharge activity was contributing to two-tone responses.

\section{Results}

Extracellular single unit responses to single- and twotone stimuli were obtained from $78 \mathrm{mid}$ frequency AVCN neurons $(\mathrm{CF}=1.5-10.0 \mathrm{kHz})$ in developing gerbils. The $\mathrm{CF}$ range studied corresponds to the basilar membrane region of greatest sensitivity in gerbil, a region for which the place code does not appear to change over the ages studied (Arjmand et al., 1988; Sanes and Rubel, 1988; Sanes et al., 1989; Rübsamen, 1992). All the neurons included in this study displayed 'primary-like' post-stimulus time histograms (PSTHs) in response to tone burst stimuli (Pfeiffer, 1966). Neurons recorded in animals older than 14 dab generally displayed monotonic rate-intensity functions for tones presented at $\mathrm{CF}$ (monotonic was defined as having no more than a $20 \%$ rate decrease with increasing input intensity). In the youngest animals (13 and 14 dab), responsiveness was quite variable and the maximum firing rates were low, consistent with a previous report of cochlear nucleus neuron discharge rate variability in young kittens (Fitzakerley et al., 1991). At these ages, roughly half of the neurons showed non-monotonicity by our criteria, which seemed to be unrelated to the first appearance of two-tone suppression (see below). All neurons displayed receptive field patterns in response to pure-tone stimuli that were devoid of inhibitory regions within the excitatory area. In terms of the classification scheme proposed by Evans and Nelson (1973) and extended by Young (1984), receptive field patterns were Type I (displaying spontaneous activity and no inhibitory side-bands), or Type III (displaying inhibitory side-bands flanking the excitatory area). We also included neurons with low spontaneous discharge activity $(<5 / \mathrm{s})$, for which the presence of side-bands is not usually detectable with conventional extracellular recording techniques. These neurons are defined here as 
Type I/III. Inhibitory side-bands of Type III neurons (in response to single-tone stimuli) always occurred at intensities 10 to $20 \mathrm{~dB}$ above $2 \mathrm{TS}$ thresholds, and the magnitude of inhibition in the side-band regions of Type III neurons was small compared to 2TS magnitudes. The types of receptive field patterns exhibited by neurons in each particular age group are given in Table 1.

Results are presented first for probe-tones placed at a level determined empirically to elicit maximal 2TS. This probe level was on average $6.4 \mathrm{~dB}$ above the excitatory area threshold (S.D. $=7.5)$. Probe levels more than about $10 \mathrm{~dB}$ below excitatory thresholds resulted in a response identical to the single-tone response: For Type I and Type I/III neurons, 2TS areas disappeared. For Type III neurons, weak inhibitory side-bands were present, the dimensions of which were the same as those recorded in the single-tone response. These areas were excluded from the subsequent analyses.

\subsection{Effects of age on maximal $2 T S$ responses}

It is a widely held belief that given sufficient recording time, 2TS can always be elicited from auditory nerve fibers recorded in adult animals (Sachs and Kiang, 1968; Schmiedt, 1982; Prijs, 1989). We found that AVCN neurons recorded from adult gerbils always displayed 2TS. In developing gerbils, 2TS was first observed in 5 out of 16 neurons (31\%) in the 14 dab age group. The subgroup of 14 dab neurons which displayed 2TS included each of the three receptive field pattern types; two neurons were Type I, one was Type III, and two were Type I/III. Rate-intensity functions measured in two out of five neurons with $2 \mathrm{TS}$ at $14 \mathrm{dab}$ were monotonic. The other three were non-monotonic by our criteria. The few neurons presented with two-tone stimuli in a 13 dab animal did not have measurable 2TS. However, the output limit of the suppressor stimuli was approximately 10 to $15 \mathrm{~dB}$ below excitatory area thresholds recorded at that age. Therefore, suppressor sound pressure levels may have been inadequate for examining $2 \mathrm{TS}$ in the $13 \mathrm{dab}$ age group. In gerbils older than 14 dab, every neuron examined exhibited 2TS given cell holding times were sufficient to present a large range of two-tone stimuli.

\subsubsection{Suppressor-tone effects}

The effects of varying suppressor-tone frequency and sound pressure level on 2TS, when present, were consistent among neurons with different receptive field types and did not appear to change with age. Representative examples of excitatory areas (diagonal fill) and 2TS areas (grey fill) recorded in gerbils at three different ages are presented in Fig. 3. Suppression areas in AVCN neurons were tuned; i.e. the suppressor intensities required to produce $2 \mathrm{TS}$ were lowest and $2 \mathrm{TS}$ magnitudes were greatest for suppressor frequencies that were relatively close to the excitatory $\mathrm{CF}$. We use the term 'best suppressor frequency' (BSF) to refer to that suppressor-tone which at the lowest intensity, produced 2TS. Suppressors lower in frequency than the BSF closely bordered or slightly overlapped the excitatory response area. Increasing the sound pressure level of the suppressor-tone generally increased the magnitude of 2TS. The largest reductions in probe-generated activity were produced by BSF suppressors presented near the maximum output level of the system (105 dB SPL).

\subsubsection{TS magnitude}

One way to evaluate potential age-related changes in the strength of $2 \mathrm{TS}$ is to examine the maximum discharge rate reduction caused by high level suppressors. Fig. 4A shows driven discharge rates measured in the presence of the probe only (open circles) and the probe together with a high level BSF suppressor (filled circles). The difference between probe only and probe plus suppressor-tone responses is referred to here as absolute 2TS magnitude. Data are shown as a function of age and neuron receptive field type is indicated by the different symbols. The driven discharge rates presented in this figure have been normalized with respect to spontaneous activity. That is, spike rates for the probe-alone condition have had spontaneous rate subtracted out,

Table 1

Group information

\begin{tabular}{lllcccc}
\hline Age $(\mathrm{dab})$ & Mean CF (S.D.) & Total animals & Type I neurons & Type III neurons & Type I/III neurons & Total neurons \\
\hline 13 & $2.68(0.54)$ & 1 & 5 & 0 & 6 & 11 \\
14 & $2.51(0.91)$ & 4 & 5 & 1 & 6 & 16 \\
$15-17$ & $3.49(2.4)$ & 6 & 3 & 11 & 5 & 14 \\
$18-21$ & $3.48(1.7)$ & 8 & 0 & 14 & 8 & 16 \\
$23-60$ & $2.84(1.4)$ & 11 & 0 & 31 & 34 & 78 \\
Total & $3.00(1.6)$ & 30 & 13 &
\end{tabular}

Mean characteristic frequency (CF), number of animals, and number of neurons in a given age group where age is given in days after birth (dab). The breakdown of neurons by the receptive field pattern expressed is shown for each age group (e.g. Type I, Type III, and Type I/III). Type I neurons are devoid of inhibition in their response areas and have spontaneous discharge rates ( $\geq 5$ spikes/second). Inhibitory side-bands flank the excitatory areas of Type III neurons. Type I/III neurons possess low spontaneous discharge rates $(<5$ spikes/second) which prevent them from being unambiguously classified as either Type I or Type III neurons. 

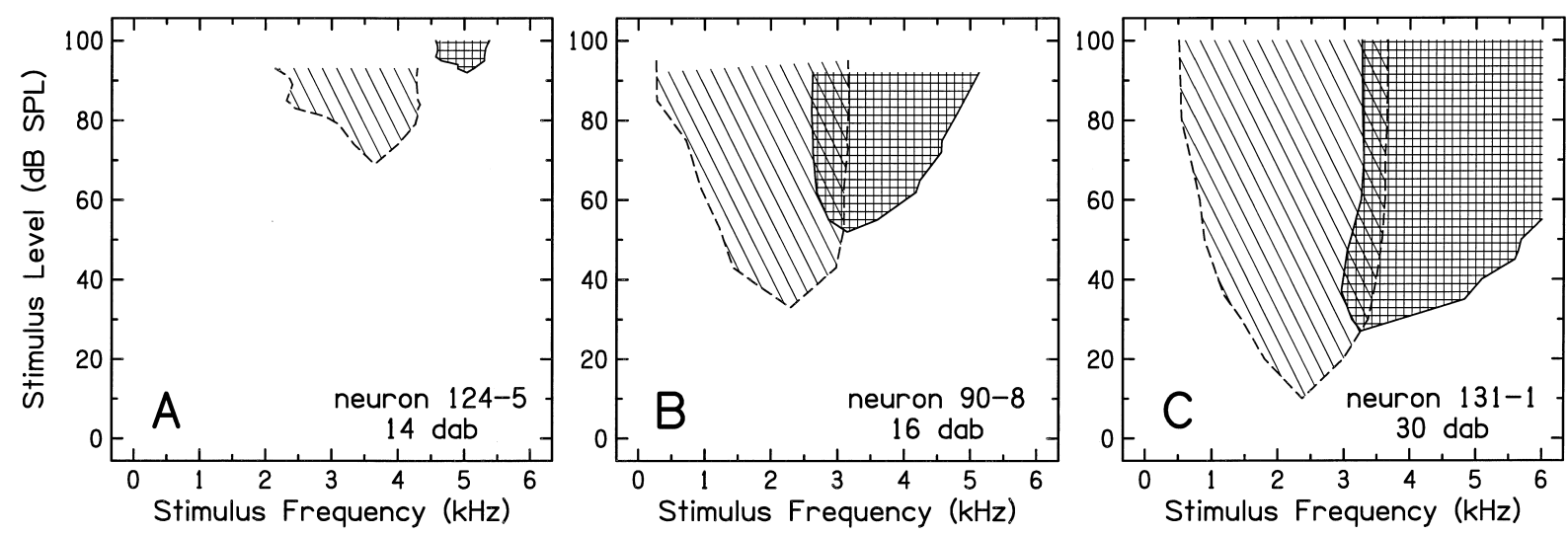

Fig. 3. A comparison of 2TS areas (grey fill) and excitatory areas (diagonal fill) at three different ages. Suppression areas are shown superimposed on the excitatory area recordings. For 2TS areas, the ordinate and abscissa indicate the level and frequency of the suppressor-tone needed to produce a criterion decrease in the response to the fixed level CF probe-tone. For excitatory areas, the ordinate gives the level of the frequency tone (shown on the abscissa) required to generate a criterion amount of excitation above the spontaneous activity level.

and values for the two-tone condition (with BSF) indicate the firing rates as compared to the spontaneous firing rate.

Irrespective of the amount of excitation produced by the probe alone, high level suppressors were able to virtually eliminate driven discharge activity. Probe-driven activity was often reduced below spontaneous discharge rates, consistent with enhancement of cochlear $2 \mathrm{TS}$ by neuronal inhibition. The amount of the discharge rate reduction was positively correlated with the response rate recorded during the presentation of the probe-tone alone. Results of a multiple regression analysis using stepwise selection of independent variables produced a model in which the normalized probe rate together with the spontaneous rate, accounted for $98 \%$ of the variability on 2 TS magnitude $\left(r^{2}=0.976\right)$.

A multivariate ANOVA was performed in order to examine the effects of age on the various aspects of $2 \mathrm{TS}$ of interest for this study. Significant age effects were further analyzed using appropriate univariate tests of significance. The same method of analysis was applied to features of the single-tone response. Means, standard deviations, and median values of analyzed responses are presented as a function of age in Tables 2 and 3. Results showed that there was a significant increase in absolute 2TS magnitude values with age $(F(3$, $48)=3.119, P<0.05)$. While there was an increase in the range of probe-driven discharge rates (Fig. 4A) and spontaneous discharge rates (shown in Fig. 5) from 14 dab to adulthood, only changes in probe-driven discharge activity were statistically significant $(F(3$, 51) $=3.451, P<0.05$ ).

Given the tendency for probe-driven discharge rates to increase with age, the proportion of the probe's response able to be eliminated by high level suppressors may be a more appropriate comparison across age
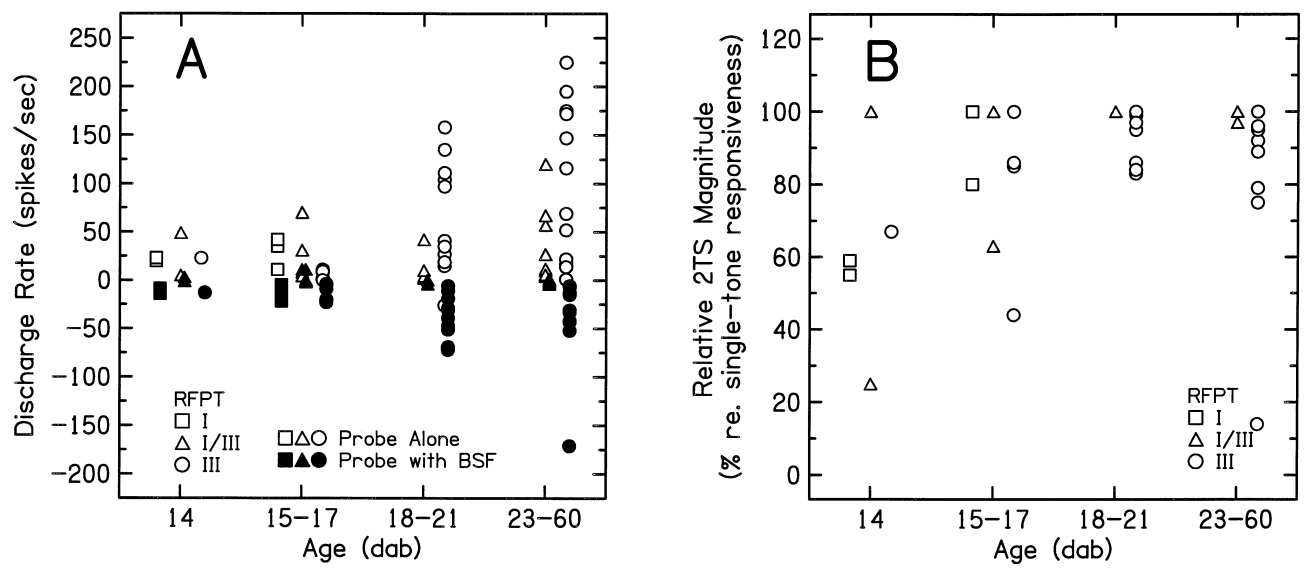

Fig. 4. A: Developmental changes in driven discharge rates and in the amount of suppression. Probe at CF alone (open symbols) and probe with BSF suppressor-tone at a high level (filled symbols). Data are normalized with respect to the spontaneous activity level. The parameter is the neuron receptive field pattern type. Absolute 2TS magnitude is the difference between discharge rates generated by the CF probe, presented alone, and together with the BSF suppressor-tone level which produced the greatest reduction in probe activity within the 2TS area. B: Relative 2TS magnitude. Data are those shown in A except here are given as the proportion of (spontaneous and driven) discharge activity collected during the presentation of the probe-tone, and able to be eliminated by a high level suppressor-tone. 


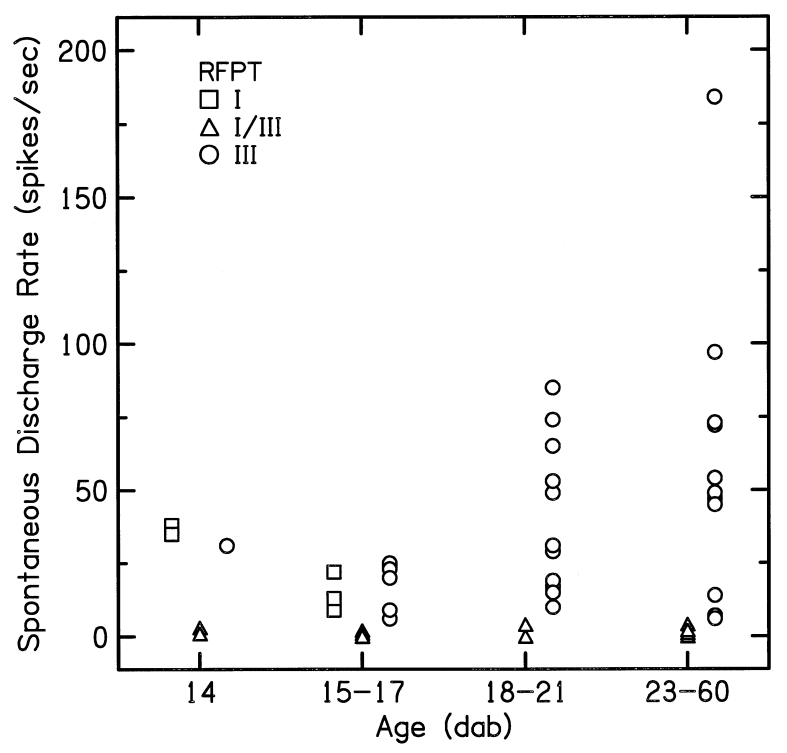

Fig. 5. Spontaneous discharge rates as a function of age. The parameter is type of receptive field pattern exhibited.

groups than absolute measures. Maximum 2TS magnitude data are shown in Fig. 4B as the percent of the probe-alone discharge rate (including spontaneous and driven activity) eliminated by the suppressor-tone. Data are plotted as a function of age and different symbols represent receptive field pattern types. For the youngest animals tested, the mean relative $2 \mathrm{TS}$ magnitude was $61 \%$. The mean increased to $90 \%$ at 15 to 17 dab, after which it remained fairly stable with age. There was a significant effect of age on relative $2 \mathrm{TS}$ magnitude values $(F(3,48)=5.102, P<0.005)$.

\subsubsection{TS threshold}

In order to begin quantifying developmental changes in the size of the regions encompassed by $2 \mathrm{TS}$ areas, the lowest intensities able to elicit 2TS (2TS thresholds) were examined across age groups. Fig. 6 shows 2TS thresholds (panel A) and excitatory thresholds at CF (panel B) as a function of age for each receptive field pattern type. Suppression thresholds decreased significantly with age $(F(3,48)=26.9, P<0.001)$ in a way that roughly paralleled developmental changes in excitatory area thresholds. Changes in excitatory area sensitivity were also statistically significant $(F(3,51)=47.7$, $P<0.001)$. There was a strong positive correlation between 2TS thresholds and excitatory thresholds $(r=0.87)$. Mean 2TS thresholds decreased from $90 \mathrm{~dB}$ SPL at 14 dab to $48 \mathrm{~dB}$ SPL at 18-21 dab. Mean excitatory thresholds at $\mathrm{CF}$ improved from $83 \mathrm{~dB}$ SPL to $16 \mathrm{~dB}$ SPL between 14 dab and adulthood. Five neurons at $14 \mathrm{dab}$ had excitatory thresholds of 47-77 dB SPL and displayed 2TS areas, which had thresholds ranging from 76 to $97 \mathrm{~dB}$ SPL. The remaining 11 neurons in the 14 dab group (not shown in Fig. 6) had excitatory thresholds of 83-95 dB SPL and did not have measurable $2 \mathrm{TS}$.

\subsubsection{TS area bandwidth}

Possible age-related changes in the size of 2TS areas were further evaluated by measuring $2 \mathrm{TS}$ area bandwidths. The width of 2TS areas was expressed in terms of 'quality' or ' $Q$ ' values in order that the suppressor frequency range associated with $2 \mathrm{TS}$ areas could be easily compared to the tuning of excitatory areas. To calculate 2TS Q10 values, the 2TS BSF was divided by the bandwidth of the 2TS area taken at $10 \mathrm{~dB}$ above the threshold of the 2TS area. Excitatory area $\mathrm{Q}$ values are equal to the excitatory $\mathrm{CF}$ divided by the bandwidth of the excitatory area taken at $10 \mathrm{~dB}$ above threshold.

Table 2

Effects of age on features of $2 \mathrm{TS}$

\begin{tabular}{|c|c|c|c|c|}
\hline & $14 \mathrm{dab}$ & 15-17 dab & $18-21 \mathrm{dab}$ & 23-60 dab \\
\hline \multicolumn{5}{|l|}{ Abs 2TS mag } \\
\hline Mean (S.D.) & $30.8(17.58)$ & $24.71(20.5)$ & $72.81(64.47)$ & $98.75(90.81)$ \\
\hline Median & 34 & 18 & 66 & 66 \\
\hline Mean (S.D.) & $61.20(26.89)$ & $89.86(17.29)$ & $96.13(6.07)$ & 90.55 (19.70) \\
\hline Median & 59 & 100 & 99.5 & 98.5 \\
\hline \multicolumn{5}{|l|}{ 2TS thresh } \\
\hline \multicolumn{5}{|l|}{ 2TS Q10 } \\
\hline Mean (S.D.) & $6.09(5.11)$ & $3.83(1.86)$ & $2.81(1.37)$ & $2.58(1.54)$ \\
\hline Median & 3.92 & 3.39 & 2.84 & 2.41 \\
\hline \multicolumn{5}{|l|}{$\mathrm{BSF} / \mathrm{CF}$ ratio } \\
\hline Mean (S.D.) & $1.34(0.26)$ & $1.37(0.14)$ & $1.5(0.31)$ & $1.61(0.29)$ \\
\hline Median & 1.27 & 1.37 & 1.43 & 1.65 \\
\hline
\end{tabular}

Relationships between two-tone suppression features and postnatal age. Features include: absolute suppression magnitude in spikes/second (Abs 2TS mag), supression magnitude taken relative to probe-alone discharge rate (Rel 2TS mag), threshold for the best suppressor frequency (2TS thresh), best suppressor frequency divided by suppression area bandwidth at $10 \mathrm{~dB}$ above 2TS threshold (2TS Q10), and the ratio of best suppressor frequency and neuron $\mathrm{CF}$ (BSF/CF ratio). 

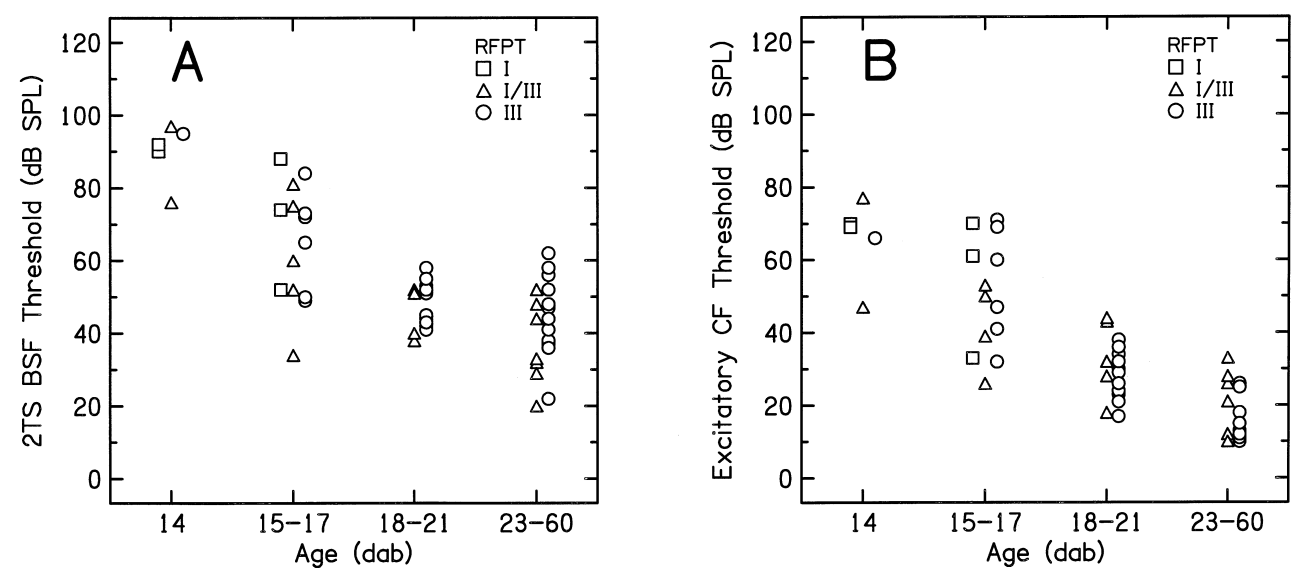

Fig. 6. A comparison of the thresholds of $2 \mathrm{TS}$ areas (panel A) and excitatory areas (panel B) for each age group. Different symbols indicate different receptive field pattern types.

Although data are limited, it appears that changes in the bandwidth of 2TS areas were subtle and occurred early in development. Fig. 7 shows 2 TS and excitatory area Q10 values plotted as a function of age (panels A and $\mathrm{B}$, respectively). There were a few relatively high 2TS area Q10 values at 14 to 17 dab. Results of an ANOVA showed a significant effect of age on $2 \mathrm{TS}$ Q10 values $(F(3,48)=4.162, P=0.01)$. However, there was not a significant effect of age on excitatory area Q10 values. Multiple regression analysis revealed a low to moderate, negative correlation between 2TS Q10 and both age category and excitatory area Q10 (overall $r=0.512$ ) (i.e. immature, broadly tuned neurons were somewhat more likely to display a relatively narrow range of effective suppressor-tone frequencies).

\subsubsection{BSF/CF ratio}

It is not known whether the 2TS BSF shifts relative to the excitatory $\mathrm{CF}$ as a function of age. The relationship between the BSF and the CF is often expressed by the ratio $\mathrm{BSF} / \mathrm{CF}$. The $\mathrm{BSF} / \mathrm{CF}$ ratio is known to de- pend on CF (Greenwood et al., 1976; Prijs, 1989), suggesting it could be influenced by the developmental changes that also affect the degree of neural tuning.

Fig. 8 shows $\mathrm{BSF} / \mathrm{CF}$ ratios for individual neurons as a function of age. The median $\mathrm{BSF} / \mathrm{CF}$ ratio increased with age $(F(3,48)=4.706, P<0.01)$, despite the fact that excitatory area tuning was not age-dependent (Fig. 7). This result would appear to contradict a hypothesis that the BSF/CF ratio is related to frequency selectivity. However, multiple regression analysis showed a low to moderate positive correlation between the $\mathrm{BSF} / \mathrm{CF}$ ratio and excitatory area Q10 values and age $(r=0.587)$, with a negative zero order correlation between the $\mathrm{BSF} / \mathrm{CF}$ ratio and excitatory area Q10 $(r=-0.511)$.

\subsection{Comparison of probe-tone level effects and age effects}

The results reported above were always collected using a probe-tone that was on average about $6 \mathrm{~dB}$ above

Table 3

Effects of age on features of single-tone responses

\begin{tabular}{|c|c|c|c|c|}
\hline & $14 \mathrm{dab}$ & $15-17 \mathrm{dab}$ & $18-21 \mathrm{dab}$ & 23-60 dab \\
\hline \multicolumn{5}{|l|}{ Spont rate } \\
\hline Mean (S.D.) & $9.25(13.06)$ & $11.07(9.89)$ & $28.19(28.54)$ & $35.1(46.46)$ \\
\hline Median & 3 & 9 & 18 & 10.5 \\
\hline Mean (S.D) & $24(15.84)$ & $17.36(20.05)$ & $48.5(54.7)$ & $75.4(73.2)$ \\
\hline Median & 23 & 10 & 31 & 54.5 \\
\hline \multicolumn{5}{|l|}{ Excit thresh } \\
\hline \multicolumn{5}{|l|}{ Excit Q10 } \\
\hline Mean & $3.01(1.0)$ & $2.38(0.79)$ & $2.35(0.74)$ & $2.26(1.01)$ \\
\hline Median & 2.83 & 2.15 & 2.32 & 2.11 \\
\hline
\end{tabular}

Relationships between features of single-tone responses and postnatal age. Features analyzed include spontaneous discharge rate (Spont rate), probe-alone-driven discharge rate normalized for spontaneous discharge activity (Probe rate), excitatory area threshold (Excit thresh), and excitatory CF divided by the bandwidth of the excitatory area taken at $10 \mathrm{~dB}$ above threshold (Excit Q10). 

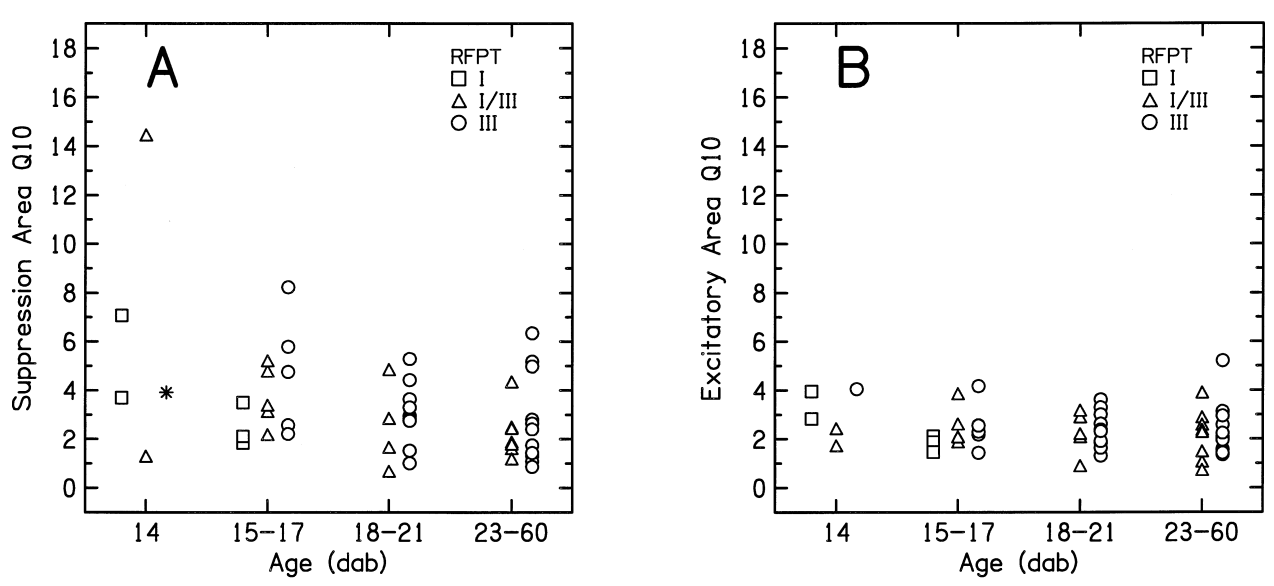

Fig. 7. A comparison of $2 \mathrm{TS}$ area Q10 values (panel A) and excitatory area Q10 values (panel B) as a function of age and for different receptive field patterns. Values were obtained by dividing the 2TS area BSF or excitatory area CF by the bandwidth measured at $10 \mathrm{~dB}$ above that area's threshold. The bandwidth of one 2TS area, recorded from a Type III neuron in a 14 dab gerbil, was extrapolated using a piecewise function consisting of a parabola and two lines fit to the iso-response curve data. The corresponding Q10 value is indicated by the star symbol.

threshold. The purpose of the experiments reported in this section was to determine whether 'immature-looking' 2TS responses could be produced by reducing the proportion of the $\mathrm{CF}$ probe's basilar membrane response caused by the so-called cochlear amplifier. Effects on AVCN 2TS caused by varying the level of the probe-tone from near threshold to up to $70 \mathrm{~dB}$ above it were examined. It was hypothesized that for a given neuron, high level probe-tones should produce proportionately less cochlear suppression than low level probetones. It was also thought that for a given neuron, contributions to the two-tone response by cochlear nucleus inhibition might increase as the probe-tone level was raised.

In Fig. 9, 2TS areas obtained using a range of probetone intensities are shown for the same three neurons introduced in Fig. 3. For each neuron, systematic increases in the intensity of the probe created 2TS areas which were qualitatively similar to those recorded in animals at consecutively earlier developmental stages.

A multifactor analysis of covariance (MANCOVA) was performed in order to determine whether features of AVCN 2TS (absolute 2TS magnitude, relative 2TS magnitude, 2TS threshold, 2TS Q10, and BSF/CF ratio) were affected by age where the level of the probetone was used as a covariate. This statistical manipulation compares ages for a given (constant) probe level. Results indicate that only absolute 2TS magnitude and 2TS threshold showed significant effects of age where probe intensity level was taken into account $(F(2$, $138)=21.402, \quad P<0.001$ and $F(2, \quad 138)=3.968$, $P<0.05$, respectively).

\subsubsection{TS magnitude}

Suppression magnitudes presented in the previous section were produced by suppressor-tones presented between about 80 and $105 \mathrm{~dB}$ SPL. Probe level effects on rate reductions caused by low and moderate level BSF suppressor-tones were also examined in a subset of neurons. Fig. 10 shows representative examples of neuronal discharge rates as a function of probe-tone intensity obtained under single-tone (open circles) and twotone (filled circles) conditions. For the two-tone conditions, BSF tones were presented at a range of (constant) sound pressure levels as indicated on the figure. Data are for the same three neurons introduced in Fig. 3, recorded in gerbils aged 14, 16, and 30 days (panels $\mathrm{A}, \mathrm{B}$, and $\mathrm{C}$, respectively).

Absolute 2TS magnitude values obtained for low to moderate level suppressors tended to remain fairly constant or decreased with increasing probe intensity (for example, see curves in Fig. 10B corresponding to sup-

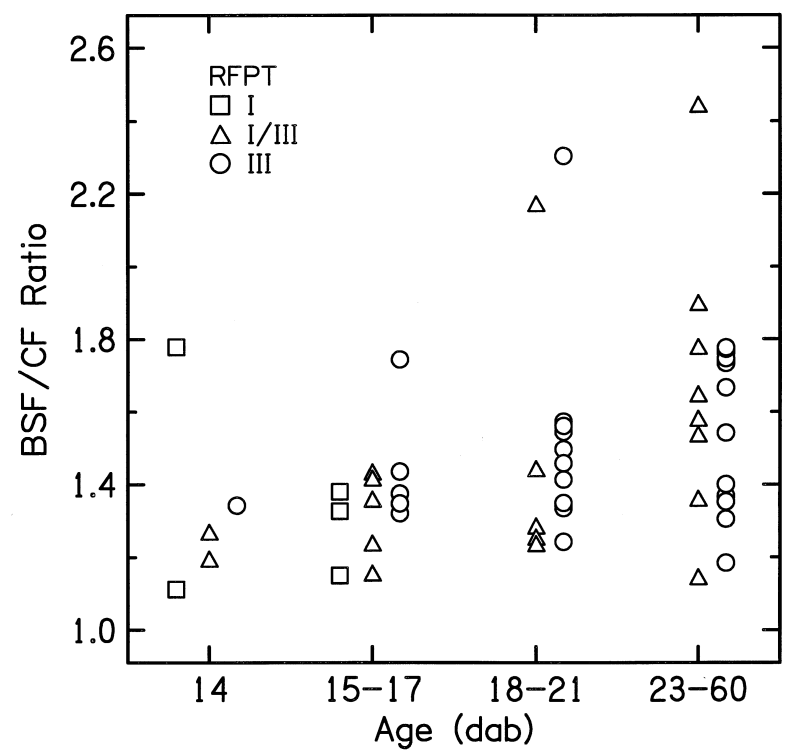

Fig. 8. Best frequency ratios as a function of age, and shown for different neuron receptive field pattern types. Values were obtained by dividing the $2 \mathrm{TS}$ area BSF by the excitatory area CF. 

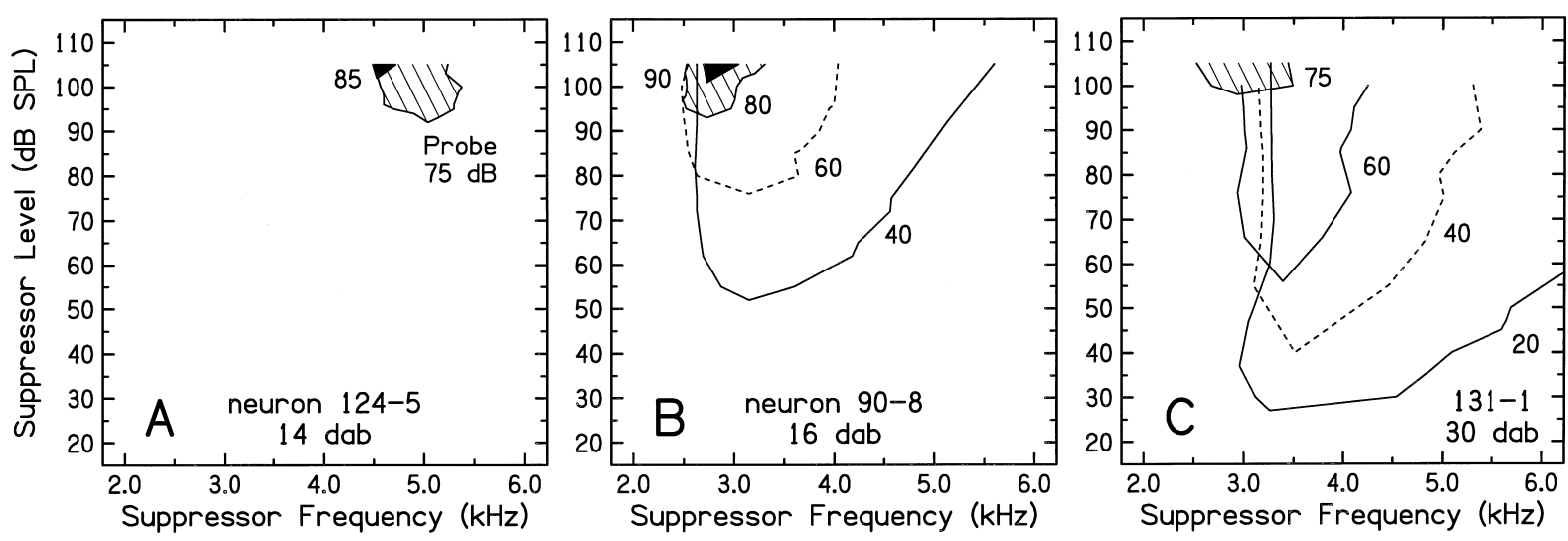

Fig. 9. Examples of 2TS areas shown for up to four probe sound pressure levels (indicated on the figure) per neuron. Panels A, B, and C show $2 \mathrm{TS}$ areas for gerbils aged 14, 16, and $30 \mathrm{dab}$, respectively. Excitatory areas have CFs of $3.7 \mathrm{kHz}$ (panel A) or $2.3 \mathrm{kHz}$ (panels B and C).

pressor levels of 40 to $80 \mathrm{~dB}$ SPL). A similar effect has been shown at the level of the auditory nerve (Abbas and Sachs, 1976; Javel et al., 1978; Costalupes et al., 1987; Sokolowski et al., 1989; Delgutte, 1990), and also the basilar membrane, where 2TS originates (Ruggero et al., 1992; Rhode and Cooper, 1993; Cooper, 1996). However, two-tone rate level functions obtained using high level suppressors, exhibited a relatively shallow slope (refer to the curves corresponding to the $100 \mathrm{~dB}$ SPL suppressors in Fig. 10B and C). Consequently, increases in the probe intensity were generally accompanied by increases in the discharge rate reductions induced by high level BSF suppressors, as shown in Fig. 11. The three panels in Fig. 11 represent three age groups (14-17, 18-21, and 23-60 dab). Since only one neuron at 14 dab displayed measurable $2 \mathrm{TS}$ at more than one probe level, these data were included with those of the 15-17 dab animals. After reaching their maxima, 2TS magnitudes rapidly diminished with further increases in the probe level.

Probe-alone discharge rate accounted for $82.7 \%$ of the variance on 2TS magnitude recorded in each given neuron for a range of probe levels $\left(r^{2}=0.827\right)$. Results of a multiple regression analysis showed that the probetone level, together with probe-alone discharge rate, contributed slightly to the overall model, raising the $r^{2}$ value to $83.2 \%$. Age did not account for any additional variance. These results indicate that even for high level probe-tones, high level suppressors were able to reduce most of the available probe-generated activity, suggesting responses were influenced by cochlear nucleus inhibition.

It appears from the data presented in Fig. 11 that rate reductions, particularly those obtained for a given high level probe-tone, tended to be greater in older animals. The results of an ANCOVA, presented above, support this conclusion (i.e., where probe level was held constant as a covariate, there was a significant effect of age on absolute 2TS magnitude). This result is probably related to the fact that older animals also tended to display relatively higher probe-alone-driven discharge rates than younger animals.
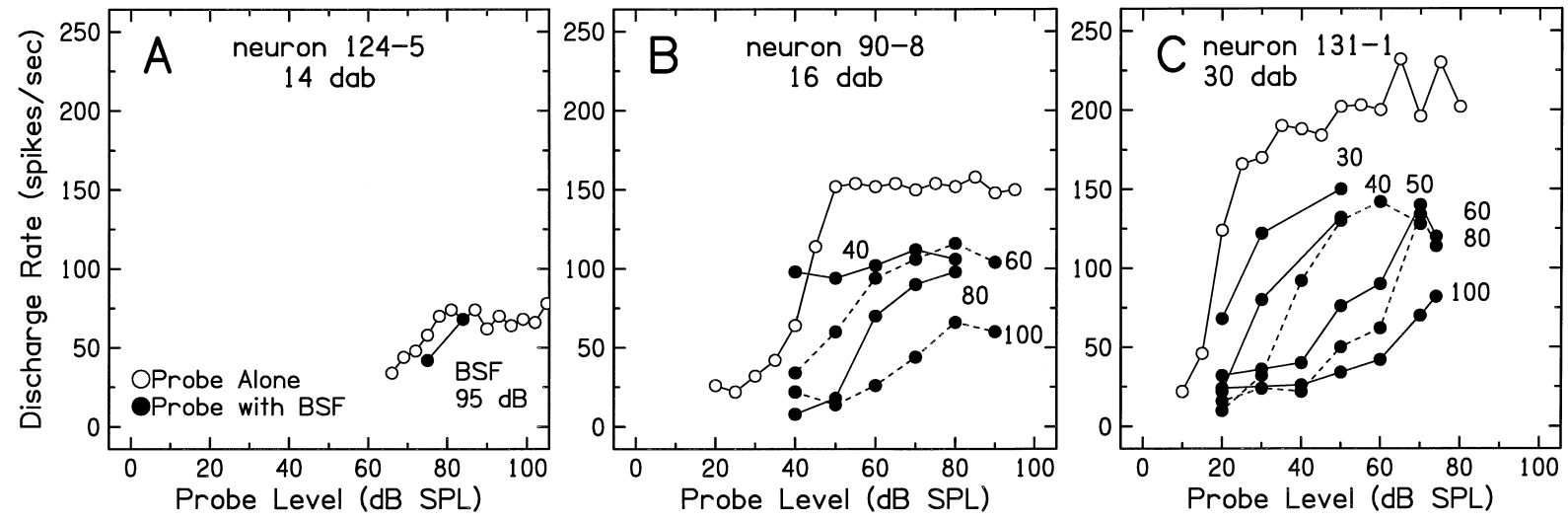

Fig. 10. Discharge rate activity is displayed as a function of probe intensity level for probe-alone (open circles) and for probe plus suppressor (filled circles) conditions in gerbils aged 14, 16, and 30 dab. The intensity of the BSF suppressor-tones is indicated in the figure. 

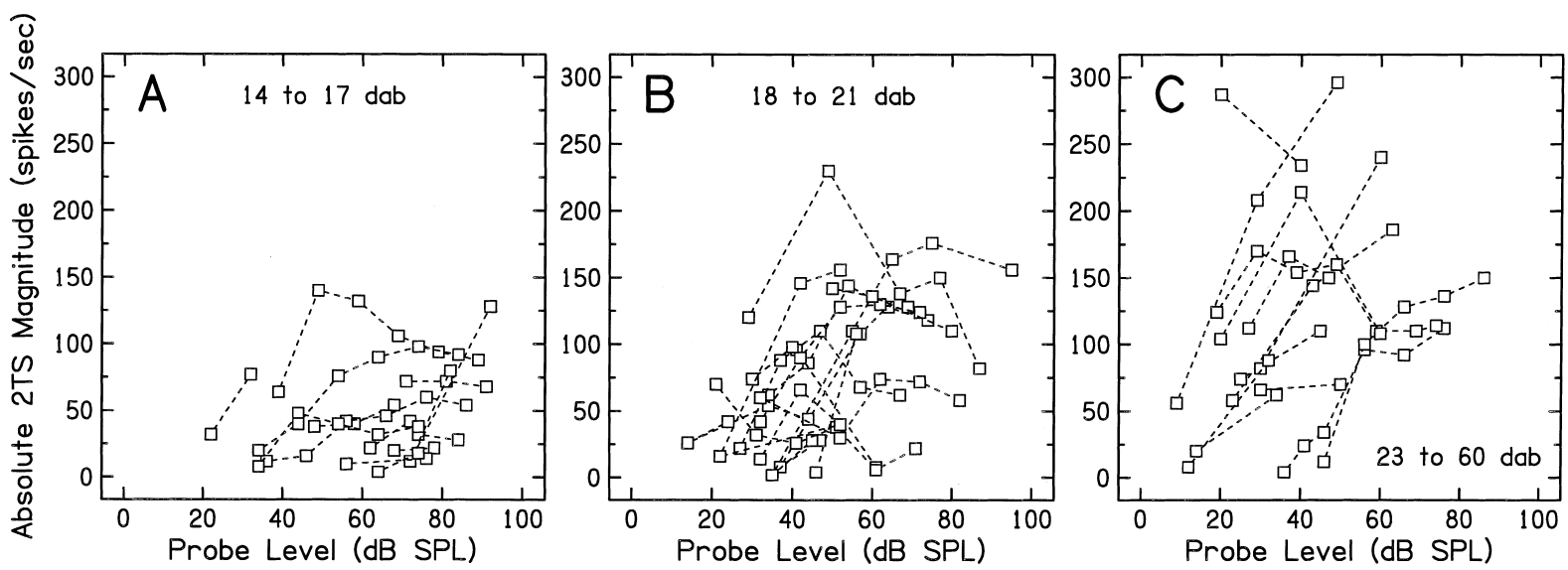

Fig. 11. Absolute 2TS magnitude (spikes/second) as a function of probe intensity level for individual neurons. Only those neurons for which suppression was exhibited at more than one probe level are shown. Each panel represents a different age group as indicated on the figure. The 14 dab data were combined with those obtained from 15 to 17 dab animals.

While the maximum absolute 2TS magnitude within a given 2TS area generally increased with increases in the probe sound pressure level, relative $2 \mathrm{TS}$ magnitude obtained for high level suppressors declined as the probe intensity was raised for the majority of neurons $(83 \%)$. Thus, level-dependent increases in absolute 2TS magnitude were apparently not proportional to changes in the probe-alone discharge rate. Relative 2TS magnitude obtained over a range of probe levels was not correlated with the level of the probe-tone or age.

\subsubsection{TS threshold}

Increasing the sound pressure level of the CF probetone has been shown to result in an upward shift of the 2TS areas recorded from auditory nerve fiber responses (Sachs and Kiang, 1968; Prijs, 1989). In the present study, there was a systematic relationship between the lowest suppressor-tone level able to elicit 2TS (2TS threshold) and probe-tone level, at all ages tested. A stepwise multiple regression analysis was performed with probe level, excitatory area threshold and age as variables. The final model showed that probe level accounted for $77 \%$ of the variance $\left(r^{2}=0.765\right)$. Neither excitatory area threshold, nor age explained any additional variability. The overall regression equation generated by the data from each of the age groups was $Y=30.35+\left(0.919^{*}\right.$ probe level $)$. A slightly larger slope value has been reported for rate suppression in adult guinea pig auditory nerve fibers (Prijs, 1989).

Similar correlations relating 2TS threshold with probe-tone intensity level were obtained across age groups $(r=0.91$ at $14-17 \mathrm{dab}, r=0.81$ at $18-21 \mathrm{dab}$, $r=0.79$ at $23-60 \mathrm{dab})$. However, the height of the regression lines calculated for individual age groups decreased with age (not shown), indicating that for a given probe-tone level, 2TS thresholds of mature animals were still somewhat lower than those of immature animals. Results of an ANCOVA (presented above), which used probe-tone level as a covariate, support these conclusions, as there was a significant effect of age even where level was held constant.

Based on the overall regression equation model relating 2TS thresholds to probe-tone level (given above), the output limits of our suppressor-tone stimuli (105 dB SPL) effectively restricted suppression measurements to probe-tones below about 85 dB SPL (108= $30.35+(0.919 * 85))$. In the seven neurons recorded in animals ranging in age from 15 to $60 \mathrm{dab}$, for which we were able to present extremely high probe intensities, probe levels incremented above 90-95 dB SPL no longer resulted in measurable $2 \mathrm{TS}$.

\subsubsection{TS bandwidth}

A multiple regression analysis showed 2TS Q10 values were not correlated with probe level, consistent with 2TS responses recorded in cat auditory nerve fibers (Abbas and Sachs, 1976). In addition, 2TS Q10 values obtained over a range of probe-tone levels were not systematically related to excitatory area Q10 or age.

\subsection{4. $B S F / C F$ ratio}

The 2TS area BSF appeared to shift to slightly lower frequencies as the intensity of the (constant frequency) probe-tone increased (i.e. at a given age, high level BSF/ $\mathrm{CF}$ ratios tended toward values obtained in neurons early in development). However, this trend was not significant, consistent with the results of Greenwood et al. (1976) for AVCN neurons in cat. While probe-tone level was dropped out of the final model, multiple regression analysis showed that the $\mathrm{BSF} / \mathrm{CF}$ ratio was negatively related to both excitatory area Q10 and age. These two variables were moderately correlated with $\mathrm{BSF} / \mathrm{CF}$ ratio $(r=0.567)$. 


\section{Discussion}

\subsection{Summary}

Our results support the following conclusions: (1) The behavior of AVCN 2TS in developing gerbils is largely due to cochlear 2TS mechanisms. (2) At all ages, two-tone responses of AVCN neurons are augmented by inhibition of central origin, at least where high level suppressors are used. (3) Developmental changes in AVCN 2TS involve an expansion of CFspecific non-linearities toward lower stimulus levels with age, while, two-tone responses to supra-threshold probe stimuli were remarkably consistent across the ages studied. (4) 2TS and neuronal sensitivity appear to be mutually related, such that $2 \mathrm{TS}$ can be used to infer the increasing contribution by the cochlear amplifier to probe-driven discharge activity over development. (5) Although its 'gain' increases postnatally, the cochlear amplifier appears to be present and fundamentally adult-like by 15 dab for regions of the cochlea coding the mid frequencies in gerbil.

\subsection{Interpretation of $A V C N$ neuron two-tone responses}

It is well known that the response of cochlear afferents and cochlear nucleus neurons to a single tone can be substantially reduced by the presentation of an additional tone or band of noise (auditory nerve: Sachs and Kiang, 1968; for more recent references see Costalupes et al., 1987; Delgutte, 1990; cochlear nucleus: early examples are Evans, 1974; Rose et al., 1974; Evans and Palmer, 1975; Greenwood et al., 1976; more recently Rhode and Greenberg, 1994; Evans and Zhao, 1995). The extent to which the cochlear mechanism $2 \mathrm{TS}$ underlies the two-tone effect in the AVCN is not known, although it has been argued that $2 \mathrm{TS}$ in neurons of the ventral and dorsal cochlear nucleus (VCN and DCN, respectively) is predominantly of the type observed in cochlear afferents (Evans and Palmer, 1975; Palmer and Evans, 1982; Gibson et al., 1985; Rhode and Greenberg, 1994; Rübsamen et al., 1998). Evans and Zhao (1995) showed that response reductions caused by two-tone stimulation in Type I/III neurons of the DCN could be partially eliminated by iontophoretic application of the glycine receptor agonist, strychnine (the application of biccuculine had no effect). They concluded that 2TS in DCN Type I/III neurons was caused by both cochlear 2TS and cochlear nucleus inhibition. Rübsamen et al. (1998) recorded exclusively from AVCN Type I neurons displaying waveforms which contained a clear prepotential preceding a larger amplitude spike response. By adjusting the height of the response trigger, the prepotential response, which is thought to be generated presynaptically by the auditory nerve (e.g. Pfeiffer, 1966), could be measured in relation to the discharge activity of the (postsynaptic) AVCN cell. Using this method, it was shown that 2TS areas of AVCN neurons were more pronounced versions of their auditory nerve input in terms of the overall size of the regions, and the magnitude of response reductions. These results demonstrate that cochlear 2TS is enhanced by neuronal inhibition at the level of the AVCN. Our data, which are comprised of single unit responses recorded from Type I, Type I/III, and Type III AVCN neurons are also consistent with the twotone effect measured in the AVCN having a dual origin.

The main focus of this investigation was to examine the ontogeny of two-tone responses in primary-like neurons of the AVCN. All neurons included in our study lacked obvious inhibition affecting the responses to tones presented within the excitatory area of their receptive fields, suggesting that central processing of the auditory nerve input to these neurons was minimal.

Consistent with the literature, most aspects of 2TS behavior observed in the present study resembled cochlear 2TS. The most notable exception was with regard to the maximum rate reductions produced under twotone stimulation. High level suppressors were often able to cause discharge rate reductions to below spontaneous rate levels. This was true across all age groups. Lower magnitudes of suppression are generally reported for auditory nerve fibers (Fahey and Allen, 1985; Costalupes et al., 1987; Delgutte, 1990). For example, Fahey and Allen (1985) reported that the driven rate of high frequency auditory nerve fibers was routinely reduced to spontaneous rate for suppressors presented below CF, but never for above CF suppressors, or for low frequency fibers.

The mean $\mathrm{BSF} / \mathrm{CF}$ ratio for mature $\mathrm{AVCN}$ neurons in our data (1.5) was slightly large compared to the commonly reported value for auditory nerve fibers which is closer to 1 (cat: Javel et al., 1978; chinchilla: Costalupes et al., 1987; gerbil: Schmiedt, 1982; cat and guinea pig: Cooper and Rhode, 1993). Few studies have systematically examined $\mathrm{BSF} / \mathrm{CF}$ ratio in cochlear nucleus. Greenwood et al. (1976) found that BSF/CF ratios decreased with increasing $\mathrm{CF}$ frequency such that the relationship remained essentially constant when converted to distance along the basilar membrane in cat. The mean BSF/CF ratio reported in that study was $0.66 \mathrm{~mm}$ which corresponds to $\mathrm{BSF} / \mathrm{CF}$ ratios of approximately 1.24 to 1.16 for $\mathrm{CF}$ frequencies ranging from about 0.75 to $7.0 \mathrm{kHz}$. Differences in BSF/CF ratios across studies might be related to the different species used, or the relatively high $2 \mathrm{TS}$ thresholds in the present study which were on average about $15 \mathrm{~dB}$ above the tip of the excitatory tuning curve. The latter observation suggests that our statistical criterion for determining 2TS may have been conservative compared to that used in other studies. 


\subsection{Development of responses to single-tone stimuli}

Auditory nerve fibers and AVCN neurons first respond to a restricted range of mid to low frequency stimuli. These early responses require high input intensities (approximately $120 \mathrm{~dB}$ SPL) and are broadly tuned compared to those obtained in mature animals. In addition, response thresholds improve systematically with age. Some investigators contend that tuning develops gradually (e.g. Romand, 1983) and others have shown that the responses of relatively high frequency auditory nerve fibers (in gerbil, above about $4 \mathrm{kHz}$ ) display adult-like tuning early in development, at the time when neuronal tip regions are first able to be identified (gerbil: Woolf and Ryan, 1985; cat: Walsh and McGee, 1990). Consistent with previously published accounts, the activity generated by AVCN neurons in the gerbil changed dramatically over the first three weeks of life. Neural tuning did not change significantly from 14 to $30 \mathrm{dab}$, while sensitivity improved gradually with age. The range of driven discharge rates in response to low level CF probe-tones increased with age, as did the range of spontaneous discharge rates observed for a given age group.

\subsection{Development of responses to two-tone stimuli}

Suppression was first observed at 14 dab in the $61 \%$ of neurons at that age which displayed excitatory area thresholds under about $90 \mathrm{~dB}$ SPL. The appearance of $2 \mathrm{TS}$ roughly coincided with the time when DPOAEs could first be measured in the gerbil (Norton et al., 1991; Mills et al., 1994, Mills and Rubel, 1996). Suppression was not expected to be present at 13 dab when driven discharge activity required stimulus levels which were beyond the output limits of the suppressor-tone (105 dB SPL), and the endocochlear potential is severely reduced relative to adults (Woolf et al., 1986; McGuirt et al., 1995).

Suppression was routinely measured after 14 dab. The ubiquity of 2TS in gerbils as young as $15 \mathrm{dab}$ contradicts a previous study in young kittens (Fitzakerley et al., 1994a, Fitzakerley et al., 1994b). These investigators report that while 2TS was correlated with age, it could be elicited from only a subgroup of cochlear afferents and primary-like AVCN neurons after its initial appearance at 69 and 73 days gestational age. The percentage of neurons recorded which exhibited 2TS in adult cats for the $\mathrm{CF}$ ranges $0.85-2.75,2.75-7.96$, and $7.96-21.5 \mathrm{kHz}$, was $33 \%$, $23 \%$, and $85 \%$, respectively (Fitzakerley et al., 1994b). Some of the neurons which failed to exhibit 2TS possessed low thresholds and a high degree of tuning, suggesting the relatively limited number of two-tone stimulus combinations and the conservative criterion for the presence of 2TS employed by Fitzakerley and colleagues may have caused $2 \mathrm{TS}$ to be missed in some instances.

In the current study, the probe level at which responsiveness was maximally affected by suppressor-tones was close to excitatory area thresholds (mean was 6.4 $\mathrm{dB}$ re: excitatory area threshold, S.D. =7.5). This is consistent with reports of auditory nerve discharge rate suppression (e.g. Abbas and Sachs, 1976; Sachs and Kiang, 1968). When suppression of these low level probe-driven responses was compared across age groups, there was a constant relationship between the lowest suppressor-tone level able to cause 2TS (2TS threshold) and excitatory area thresholds measured at a given time during development $(r=0.93)$. That is, $2 \mathrm{TS}$ threshold development paralleled improvements in excitatory area sensitivity. There was also a strong correlation between the strength of 2TS rate reductions (absolute 2TS magnitude) and the discharge activity collected during stimulation by the probe-tone alone $(r=0.98)$. Both showed an age-related increase. The bandwidth of 2TS areas (2TS Q10) appeared to increase from $14 \mathrm{dab}$ to 15 to $17 \mathrm{dab}$. There was a low to moderate correlation between 2TS Q10 and both age and excitatory area tuning together $(r=0.512)$. Separately, each predictor was inversely related (negatively correlated) to 2TS Q10. There was also an age-related increase in the frequency separation between the best suppressor frequency (BSF) and the excitatory area CF. Consequently, the BSF/CF ratio increased with age. The BSF/CF ratio was correlated with age and excitatory area $\mathrm{Q}$ values $(r=0.587)$; the zero order correlation between $\mathrm{BSF} / \mathrm{CF}$ ratio and excitatory area Q10 was negative. Taken together, these results demonstrate a strong interrelationship between neuronal responses to single- and two-tone stimuli during early postnatal development in gerbil. This finding is consistent with observations in adult animals in which $\mathrm{OHC}$ system damage simultaneously affected 2TS and cochlear sensitivity and/or tuning (basilar membrane: Ruggero et al., 1992; auditory nerve: Robertson, 1976; Dallos et al., 1980).

\subsection{Effect of probe level on two-tone responses}

Fitzakerley et al. (1994a) demonstrated that one of the most important factors influencing 2TS magnitude in developing and mature animals was the level of the CF probe-tone. We extended this finding by examining 2TS for up to eight probe levels per neuron, and by comparing level-dependent changes in 2TS across age groups. Due to developmental changes in the middle ear (Woolf et al., 1986), and in passive cochlear mechanics as inferred from DPOAE investigations (Mills et al., 1994; Mills and Rubel, 1996), a given probe presentation level probably does not yield exactly the same level within the cochleas of animals at different 
ages. However, it is likely that for a given neuron, the probe-tone level influences the amount of travelling wave amplification invoked at $\mathrm{CF}$.

Basilar membrane studies have shown that the relative contribution of cochlear amplifier gain to the response is diminished as the input amplitude is increased, regardless of whether the displacement at $\mathrm{CF}$ is caused by one or two tones. The proportion of the basilar membrane's response eliminated following the administration of furosemide is greatest for low level signals (Ruggero and Rich, 1991). Like furosemide intoxication, cochlear 2TS involves a reduction of the active amplification of the probe's response on the basilar membrane and is greatest for low level probe-tones (e.g. Ruggero et al., 1992; Rhode and Cooper, 1993; Cooper, 1996). In contrast to $2 \mathrm{TS}$ originating in the cochlea, the magnitude of neural inhibition affecting two-tone responses in the AVCN might be expected to increase as the level of either of the stimulating tones are raised.

Six Type III neurons excluded from the present data set because they displayed strongly non-monotonic rate-intensity functions, and in some cases also had 'chopper' post-stimulus time histograms, responded to increases in the probe-tone level in the manner predicted. For these neurons, increasing the level of the probe-tone increased the size of the inhibitory regions, resulting in areas of inhibition that extended over the excitatory area defined under single-tone stimulation. The primary-like neurons whose two-tone responses are reported here also generally behaved as expected with respect to changes in the probe-tone intensity. Those features of AVCN 2TS that changed with age were in general also influenced by the presentation level of the probe-tone. Consequently, for a given neuron, raising the probe-tone intensity caused the two-tone response to resemble $2 \mathrm{TS}$ obtained at earlier developmental stages.

\subsection{Development of the cochlear amplifier}

The gerbil middle ear system is thought to be functional and mostly clear of mesenchyme by approximately 16 days after birth (dab) (Finck et al., 1972; Woolf et al., 1986). Responses in 13 to 14 dab gerbils were probably substantially influenced by middle ear immaturities, while improvements in single- and twotone responses observed after about 16 dab were probably largely related to changes in cochlear and neural processes (for review see Rübsamen, 1992; Walsh and Romand, 1992; Rübsamen and Lippe, 1998).

The development of those cochlear response properties which are likely to be produced primarily by maturation of the so-called 'cochlear amplifier' has been examined using 2TS and distortion product otoacoustic emissions (DPOAEs). These response properties appear to be byproducts of the CF-specific basilar membrane non-linearity thought to be related to $\mathrm{OHC}$ induced amplification of the travelling wave response. Fitzakerley et al. (1994a), Fitzakerley et al. (1994b) found that immature (broadly tuned and high threshold) cochlear afferents and AVCN neurons were devoid of 2TS. Suppression was first observed in two neurons having mid to low CFs (between 0.85 and $7.96 \mathrm{kHz}$ ) and mature tuning sharpness.

Like 2TS, DPOAEs are absent or small in amplitude at hearing onset (rat: Henley et al., 1989; Lenoir and Puel, 1987; gerbil: Norton et al., 1991; Mills et al., 1994). Emissions could first be elicited by mid frequency stimuli (Lenoir and Puel, 1987; Henley et al., 1989; Norton et al., 1991; Mills et al., 1994; Mills and Rubel, 1996), and later for lower frequency tones (Norton et al., 1991; Mills et al., 1994). Mills et al. (1994) and Mills and Rubel (1996) measured DPOAEs before and after the administration of furosemide in gerbils aged 14 dab to adulthood. The DPOAE component sensitive to blocking the endocochlear potential with furosemide was operationally defined as the 'active' portion of the emission. Active emissions elicited by low frequency stimuli $(0.5$ and $1.0 \mathrm{kHz})$ showed a substantial increase in amplitude with age. However, increases in emissions evoked by mid frequency stimuli ( 2.0 to $16.0 \mathrm{kHz}$ ) were comparatively small. These authors concluded that for the mid frequency range, the cochlear amplifier may be present and fundamentally adult-like by about 14 dab in gerbil.

The data presented by Mills and colleagues are consistent with the preponderance of evidence that cochlear structural development progresses from the mid base of the basilar membrane toward the apex and the extreme base (Rubel, 1978; Arjmand et al., 1988; Sanes and Rubel, 1988; Sanes et al., 1989; Rübsamen, 1992). It is likely that, similar to DPOAEs, the development of 2TS is graded across the length of the basilar membrane, warranting investigations of $2 \mathrm{TS}$ in species-specific low and high frequencies.

To the extent that outer hair cell motility is responsible for the cochlear non-linearities underlying cochlear amplifier function, the view that the structural organization of the cochlear amplifier is nearly adult-like for mid frequencies by about 14 to 15 dab in gerbil is supported by another recent study. Motile responses of gerbil OHCs isolated from the basal and middle turns attain mature thresholds in response to electrical stimulation at 12 dab (He et al., 1994). He and colleagues demonstrated that the amplitude of $\mathrm{OHC}$ oscillations relative to the $\mathrm{OHC}$ length at a given age was the same as in adults by 14 dab. The absolute amplitude of electrically evoked changes in $\mathrm{OHC}$ length attained adult-like values at 16-17 dab, when the length of the OHCs themselves reached maturity.

The present study supports the hypothesis that the 
cochlear amplifier functions as early as 14 dab in the mid frequency region of the gerbil cochlea, and that its contribution to response properties increases slightly over the subsequent 7 to 10 days. This increase is temporally correlated with the development of the endocochlear potential. It is known that in gerbils, the endocochlear potential measured along the length of the cochlea increases dramatically from about $25 \mathrm{mV}$ at $13 \mathrm{dab}$, to about $40 \mathrm{mV}$ at $15 \mathrm{dab}$ (McGuirt et al., 1995), the age when 2TS was regularly obtained in AVCN neurons. The amplitude of the EP reaches nearly adult values of about $80 \mathrm{mV}$ at $18-21$ dab, the time when excitatory and suppression area thresholds reached maturity at the frequencies studied here.

Several other factors contribute to the development of mature auditory nerve responses (Rübsamen and Lippe, 1998). Thresholds of whole nerve action potentials (CAPs) recorded from the auditory nerve in gerbil have been shown to display a rapid developmental time course compared to CAP amplitudes (McGuirt et al., 1995; Huang et al., 1995). While CAP thresholds were strongly correlated with the EP in developing gerbils, CAP amplitudes were not. This dichotomy may be related to differences in the contribution of developing neural synaptic mechanisms to the establishment of mature CAP thresholds and amplitudes (McGuirt et al., 1995). The magnitudes of probe-alone-driven discharge activity and suppressor-tone induced response reductions are among those features examined in the present study that may have been strongly influenced by the maturation of neural synapses.

It was not clear from our data sample whether contributions by central inhibitory mechanisms to two-tone responses changed with age. We found evidence of inhibition affecting at least the magnitudes of response reductions produced by high level suppressor-tones as early as $2 \mathrm{TS}$ was able to be elicited (14 dab). To our knowledge there are no studies that specifically address the physiological development of inhibition in the AVCN. However, non-monotonic rate-intensity functions may indicate that responses are influenced by central inhibitory circuits. A study that surveyed VCN neurons exhibiting a range of response patterns found no evidence for a developmental sequence in the number of neurons exhibiting non-monotonic rate level functions in gerbil (Woolf and Ryan, 1985).

\section{Acknowledgments}

This research was supported by DFG Ru 390/12-1, DFG Ru 390/13-1, NIH DC00033, and NIH DC00395. The authors wish to thank Brandon Warren for his technical assistance, and Wendy Harrison for help with the figures. We also thank Dr. Susan J. Norton and three anonymous reviewers for their helpful suggestions on earlier versions of this manuscript.

\section{References}

Abbas, P.S., Sachs, M.B., 1976. Two-tone suppression in auditory nerve fibers: Extension of a stimulus-response relationship. J. Acoust. Soc. Am. 59, 112-122.

Arjmand, E., Harris, D., Dallos, P., 1988. Developmental changes in frequency mapping of the gerbil cochlea: comparisons of two cochlear locations. Hear. Res. 32, 93-96.

Bosher, S.K., Warren, R.R., 1971. A study of the electrochemistry and osmotic relationships of the cochlear fluids in the neonatal rat at the time of the development of the endocochlear potential. J. Physiol. Lond. 212, 739-761.

Cooper, N.P., 1996. Two-tone suppression in cochlear mechanics. J. Acoust. Soc. Am. 99, 3087-3098.

Cooper, N.P., Rhode, W.S., 1993. Two-tone suppression and distortion production on the basilar membrane in the hook region of cat and guinea pig cochlea. Hear. Res. 66, 31-45.

Costalupes, J.A., Rich, N.C., Ruggero, M.A., 1987. Effects of excitatory and non-excitatory suppressor tones on two-tone rate suppression in auditory nerve fibers. Hear. Res. 26, 155-164.

Dallos, P., Harris, D.M., Relkin, E. and Cheatham, M.A. (1980) Two-tone suppression and intermodulation distortion in the cochlea: Effect of outer hair cell lesions. In: van den Brink, G. and Bilsen, F.A. (Eds.), Psychophysical, Physiological and Behavioural Studies in Hearing. Delft University Press, The Netherlands, pp. 242-249.

Delgutte, B., 1990. Two-tone rate suppression in auditory nerve fibers: dependence on suppressor frequency and level. Hear. Res. 49, 225-246.

Echteler, S.M., Arjmand, E., Dallos, P., 1989. Developmental alterations in the frequency map of the mammalian cochlea. Nature 341, 147-149.

Evans, E.F. (1974) Auditory frequency selectivity and the cochlear nerve. In: Zwicker, E. and Terhardt, E. (Eds.), Facts and Models in Hearing. Springer-Verlag, Heidelberg, pp. 118-129.

Evans, E.F., Nelson, P.G., 1973. On the functional relationship between the dorsal and ventral divisions of the cochlear nucleus of the cat. Exp. Brain Res. 17, 428-442.

Evans, E.F., Palmer, A.R., 1975. Responses of units in the cochlear nerve and nucleus of the cat to signals in the presence of bandstop noise. J. Physiol. 252, 60-62P.

Evans, E.F. and Zhao, W. (1995) Intensity coding: Dynamic range shifts in cochlear nerve and nucleus. In: Advances in Hearing Research. World Scientific Publishing Co., Singapore, pp. 233240.

Fahey, P.F., Allen, J.B., 1985. Nonlinear phenomena as observed in the ear canal and at the auditory nerve. J. Acoust. Soc. Am. 77, 599-612.

Fernandez, C., Hinojosa, R., 1974. Postnatal development of endocochlear potential and stria vascularis in the cat. Acta Otolaryngol. 78, 172-186.

Finck, A., Schneck, C.D., Hartman, A.F., 1972. Development of cochlear function in the neonate Mongolian gerbil (Meriones Unguiculatus). J. Comp. Physiol. Psych. 78, 375-380.

Fitzakerley, J.L., McGee, J., Walsh, E.J., 1991. Variability in discharge rate of cochlear nucleus neurons during development. Soc. Neurosci. Abstr. 17, 304.

Fitzakerley, J.L., McGee, J., Walsh, E.J., 1994a. Responses of peripheral auditory neurons to two-tone stimuli during development: I. Correlation with frequency selectivity. Hear. Res. 77, 135-149.

Fitzakerley, J.L., McGee, J., Walsh, E.J., 1994b. Responses of peripheral auditory neurons to two-tone stimuli during development: 
II. Factors related to neural responsiveness. Hear. Res. 77, 150161.

Geisler, C.D., Yates, G.K., Patuzzi, R.B., Johnstone, B.M., 1990. Saturation of outer hair cell receptor currents causes two-tone suppression. Hear. Res. 44, 241-256.

Gibson, D.J., Young, E.D., Costalupes, J.A., 1985. Similarity of dynamic range adjustment in auditory nerve and cochlear nuclei. J. Neurophysiol. 53, 940-958.

Greenwood, D.D., Merzenich, M.M., Roth, G.L., 1976. Some preliminary observations on the interrelations between two-tone suppression and combination-tone driving in the anteroventral cochlear nucleus of the cat. J. Acoust. Soc. Am. 59, 607633.

He, D.Z.Z., Evans, E.N., Dallos, P., 1994. First appearance and development of electromotility in neonatal outer hair cells. Hear. Res. 78, 77-90.

Henley III, C.M., Owings, M.H., Stagner, B.B., Martin, G.K., Lonsbury-Martin, B.L., 1989. Postnatal development of 2f1-f2 otoacoustic emissions in pigmented rat. Hear. Res. 43, 141-148.

Huang, J.-M., Berlin, C.I., Cullen, J.K., Jr., Wickremasinghe, A.R., 1995. Development of the VIIIth nerve compound action potential evoked by low-intensity tone pips in the Mongolian gerbil. Hear. Res. 88, 14-18.

Javel, E., Geisler, C.D., Ravindran, A., 1978. Two-tone suppression in auditory nerve of the cat: Rate-intensity and temporal analyses. J. Acoust. Soc. Am. 63, 1093-1104.

Lenoir, M., Puel, J.-L., 1987. Development of 2f1-f2 otoacoustic emissions in the rat. Hear. Res. 29, 265-271.

McGuirt, J.P., Schmiedt, R.A., Shulte, B.A., 1995. Development of cochlear potentials in the neonatal gerbil. Hear. Res. 84, 52-60.

Mills, D.M., Norton, S.J., Rubel, E.W., 1994. Development of active and passive mechanics in the mammalian cochlea. Audit. Neurosci. 1, 77-99.

Mills, D.M., Rubel, E.W., 1996. Development of the cochlear amplifier. J. Acoust. Soc. Am. 100, 428-441.

Mills, J.H. and Schmiedt, R.A. (1983) Frequency selectivity, physiological and psychophysical tuning curves and suppression. In: Tobias, J.V. and Shubert, E.D. (Eds.), Hearing Research and Theory, Vol. 2. Academic Press, New York, pp. 233-336.

Neely, S.T., Kim, D.O., 1983. An active cochlear model showing sharp tuning and high sensitivity. Hear. Res. 9, 123-130.

Neely, S.T., Kim, D.O., 1986. A model for active elements in cochlear biomechanics. J. Acoust. Soc. Am. 79, 1472-1480.

Norton, S.J., Bargones, J.Y., Rubel, E.W., 1991. Development of otoacoustic emissions in gerbil: evidence for micromechanical changes underlying development of the place code. Hear. Res. 51, 73-92.

Palmer, A.R., Evans, E.F., 1982. Intensity coding in the auditory periphery of the cat: Responses of cochlear nerve and cochlear nucleus neurons to signals in the presence of bandstop masking noise. Hear. Res. 7, 305-323.

Patuzzi, R.B., Robertson, D., 1988. Tuning in the mammalian cochlea. Physiol. Rev. 68, 1010-1082.

Pfeiffer, R.R., 1966. Anteroventral cochlear nucleus: Wave forms of extracellularly recorded spike potentials. Science 154, 667-668.

Prijs, V.F., 1989. Lower boundaries of two-tone suppression regions in the guinea pig. Hear. Res. 42, 73-82.

Rhode, W.S., 1978. Some observations of cochlear mechanics. J. Acoust. Soc. Am. 64, 158-176.

Rhode, W.S., Cooper, N.P., 1993. Two-tone suppression and distortion production on the basilar membrane in the hook region of cat and guinea pig cochleae. Hear. Res. 66, 31-45.

Rhode, W.S., Greenberg, S., 1994. Lateral suppression and inhibition in the cochlear nucleus of the cat. J. Neurophysiol. 71, 493-514.

Robertson, D., 1976. Correspondence between sharp tuning and twotone inhibition in primary auditory nerve neurones. Nature Lond. 259, 477-478.
Romand, R., 1983. Development in the frequency selectivity of auditory nerve fibers in the kitten. Neurosci. Lett. 35, 271-276.

Rose, J.E., Kitzes, L.M., Gibson, M.M., Hind, J.E., 1974. Observations on phase-sensitive neurons of anteroventral cochlear nucleus of the cat: Nonlinearity of cochlear output. J. Neurophysiol. 37, 218-253.

Rubel, E.W. (1978) Ontogeny of structure and function in the vertebrate auditory system. In: Jacobson, M. (Ed.), Handbook of Sensory Physiology IX. Development of Sensory Systems. SpringerVerlag, Berlin, pp. 135-237.

Rübsamen, R., 1992. Postnatal development of central auditory frequency maps. J. Comp. Physiol. 165, 755-769.

Rübsamen, R., Kopp, C. and Dörrscheidt, G.J. (1998) Principal component analysis applied to action potentials reveal neuronal interaction in auditory brainstem nuclei. In: Palmer, A.R., Rees, Q., Summerfield, A.Q. and Meddis, R. (Eds.), Psychophysical and Physiological Advances in Hearing. Whurr Publishers, London, pp. 352-358.

Rübsamen, R. and Lippe, W.R. (1998) Functional development of the cochlea. In: Rubel, E.W., Popper, A.N. and Fay, R.R. (Eds.), Development of the Mammalian Auditory System. Springer Handbook of Auditory Research. Springer Verlag, New York, Berlin, Heidelberg, pp. 193-270.

Rübsamen, R., Mills, D.M., Rubel, E.W., 1995. Effects of furosemide on distortion product otoacoustic emissions and on neuronal responses in the anteroventral cochlear nucleus. J. Neurophysiol. 74, $1628-1638$.

Ruggero, M.A., Rich, N.C., 1991. Furosemide alters organ of Corti mechanics: Evidence for feedback of outer hair cells upon the basilar membrane. J. Neurosci. 11, 1057-1067.

Ruggero, M.A., Robles, L., Rich, N.C., 1992. Two-tone suppression in the basilar membrane of the cochlea: Mechanical basis of the auditory-nerve rate suppression. J. Neurophysiol. 68, 1087-1099.

Sachs, M.A., Kiang, N.Y-S., 1968. Two-tone inhibition in auditorynerve fibers. J. Acoust. Soc. Am. 43, 1120-1128.

Sanes, D.H., Merickel, M., Rubel, E.W., 1989. Evidence for an alteration of the tonotopic map in the gerbil cochlea during development. J. Comp. Neurol. 279, 436-444.

Sanes, D.H. and Rubel, E.W. (1988) The development of stimulus coding in the auditory system. In: Jahn, A.R. and Santos-Sacchi, J. (Eds.), Physiology of the Ear. Raven Press, pp. 431-455.

Schmiedt, R.A., 1982. Boundaries of two-tone rate suppression of cochlear-nerve activity. Hear. Res. 7, 335-351.

Schmiedt, R.A., Zwislocki, J.J., 1980. Effects of hair cell lesions on responses of cochlear nerve fibers. II. Single- and two-tone intensity functions in relation to tuning curves. J. Neurophysiol. 43, $1390-1405$.

Schmiedt, R.A., Zwislocki, J.J., Hamernik, R.P., 1980. Effects of hair cell lesions on the responses of cochlear nerve fibers. I. Lesions, tuning curves, two-tone inhibition, and responses to trapezoidal wave patterns. J. Neurophysiol. 43, 1367-1389.

Sokolowski, B.H.A., Sachs, M.B., Goldstein, J.L., 1989. Auditory nerve rate-level functions for two-tone stimuli: possible relation to basilar membrane nonlinearity. Hear. Res. 41, 115-124.

Walsh, E.J., McGee, J., 1990. Frequency selectivity in the auditory periphery: Similarities between damaged and developing ears. Am. J. Otolaryngol. 11, 23-32.

Walsh, E.J. and Romand, R. (1992) Functional development of the cochlea and cochlear nerve. In: Romand, R. (Ed.), Development of Auditory and Vestibular Systems II. Elsevier, New York, pp. 161-219.

Woolf, N.K., Ryan, A.F., 1984. The development of auditory function in the cochlea of the Mongolian gerbil. Hear. Res. 13, 131142.

Woolf, N.K., Ryan, A.F., 1985. Ontogeny of neural discharge patterns in the ventral cochlear nucleus of the Mongolian gerbil. Dev. Brain Res. 17, 131-147. 
Woolf, N.K., Ryan, A.F., Harris, J.P., 1986. Development of mammalian endocochlear potential: normal ontogeny and effects of anoxia. Am. J. Physiol. 250, R493-R498.

Yates, G.K., Johnstone, B.M., Patuzzi, R.B., Robertson, D., 1992. Mechanical preprocessing in the mammalian cochlea. Trends Neurosci. 15, 57-61.
Young, E.D. (1984) Response characteristics of neurons of the cochlear nuclei. In: Berlin, C. (Ed.), Hearing Sciences. College-Hill Press, San Diego, pp. 423-260. 